INRA Prod. Anim.,

2013, 26 (3), 249-262

\title{
Les besoins en eau d'abreuvement des bovins laitiers : déterminismes physiologiques et quantification
}

\author{
A. BOUDON ${ }^{1,2}$, H. KHELIL-ARFA 1,2, J.-L. MÉNARD 3 , P. BRUNSCHWIG ${ }^{3}$, P. FAVERDIN ${ }^{1,2}$ \\ ${ }^{1}$ INRA, UMR1348 PEGASE, F-35590 Saint-Gilles, France \\ 2 Agrocampus Ouest, UMR1348 PEGASE, F-35000 Rennes, France \\ ${ }^{3}$ Institut de l'Elevage, 9 rue André Brouard, F-49000 Angers, France
}

Courriel : anne.boudon@rennes.inra.fr

L'eau d'abreuvement représente moins de $10 \%$ de l'eau prélevée par l'agriculture dans le monde et en France, mais c'est un besoin incompressible des élevages. Les quantités journalières d'eau bue par une vache laitière sont très variables, de quelques litres avec une herbe verte en situation tempérée à plus de 120 litres avec une ration sèche. Mieux connaître les causes de ces variations et les quantifier sont des prérequis pour mieux planifier et maîtriser les quantités d'eau utilisées en élevage laitier.

Comme le souligne le texte de Corson et Doreau (2013) de ce même numéro, un critère simple pour évaluer la contribution d'un système d'élevage au risque de pénurie d'eau est sa consommation en eau bleue, c'est-à-dire en eau douce de surface ou souterraine. En élevage, cette eau comprend systématiquement l'eau utilisée par l'irrigation, l'eau d'abreuvement et l'eau utilisée pour le nettoyage, notamment du matériel de traite. En France, l'élevage bovin laitier consomme environ 38\% de l'eau (bleue) utilisée par l'agriculture hors irrigation et plus de $75 \%$ de cette eau est destinée à l'abreuvement des animaux (Carteau et al 2010). Etant donné que, aussi bien en France qu'à l'échelle mondiale, environ $90 \%$ des prélèvements d'eau par l'agriculture sont destinés à l'irrigation, l'eau d'abreuvement, toutes espèces confondues, ne représente que $0,6 \%$ de l'eau prélevée par l'agriculture à l'échelle mondiale (Steinfeld et al 2006) et $6 \%$ à l'échelle française (Carteau et al 2010). Elle ne constitue donc pas un poste de consommation pouvant faire varier de façon importante l'efficacité d'utilisation de l'eau en agriculture mais, le caractère incompressible des besoins en eau d'abreuvement en élevage et la qualité nécessaire de cette eau, fait que cet usage peut entrer en concurrence avec d'autres usages de l'eau, notamment non agricoles pendant les périodes de déficit hydrique. L'occurrence et la durée de ces épisodes pourraient augmenter de façon significative à l'avenir si on se réfère aux prévisions du GIEC (Pachauri et Reisinger 2007) et il est aussi vraisem- blable que l'étendue des zones géographiques mondiales soumises à cette problématique augmente du fait de l'augmentation de la demande mondiale pour les produits d'origine animale (Steinfeld et al 2006).

L'eau d'abreuvement peut représenter un coût économique important pour les élevages, en particulier pour ceux dont l'approvisionnement est assuré par le réseau d'eau public. Il a été montré récemment que les fuites sur les réseaux de distribution d'eau des élevages bovins laitiers pouvaient engendrer des pertes d'eau significatives (Ménard et al 2012). Une grande partie de ces fuites ne peut être détectée que suite à une estimation précise du volume nécessaire à l'abreuvement. A l'échelle française, il a été estimé que seulement 20 à $30 \%$ des élevages avaient recours à l'eau du réseau public (ANSES 2010) et il est vraisemblable que cette proportion soit assez représentative des élevages laitiers. Cependant, les règles d'établissement et d'utilisation d'eau provenant de forages privés se sont énormément durcies ces dernières années, notamment par la nécessité de disposer de compteurs pour quantifier les prélèvements et par la mise en place de redevances sur les prélèvements les plus importants même pour un usage d'abreuvement (article L213-10-9 du code de l'environnement). Outre le coût économique que cette nouvelle réglementation peut engendrer pour les élevages les plus importants, la disponibilité de ces compteurs peut aussi être un outil pour les élevages afin de contrôler que la disponibilité en eau d'abreuvement pour le troupeau est suffisante.

L'application du protocole d'estimation du bien-être animal Welfare Quality® (2009) aux élevages laitiers français a montré que le critère d'absence de soif était respecté de façon extrêmement hétérogène sur l'ensemble du territoire (De Boyer des Roches et al 2012). Pourtant, il a clairement été montré qu'une restriction de l'abreuvement a des effets immédiats sur les performances des vaches. Notamment, Burgos et al (2001) ont illustré qu'une restriction de $50 \%$ des quantités d'eau bue chez des vaches laitières entraîne en une journée une baisse de $10 \%$ de la production laitière allant jusqu'à $20 \%$ en une semaine. De ce fait, un sous-abreuvement entraîne rapidement une moindre valorisation des aliments, ce qui a des conséquences économiques importantes.

Ces faits démontrent l'ampleur des enjeux qu'il y a à mieux quantifier les besoins en eau des animaux sur les systèmes laitiers, notamment pour $i$ ) estimer les besoins incompressibles d'eau de l'élevage, notamment pendant les périodes de sécheresse où des compétitions peuvent apparaître entre les postes d'utilisation de l'eau, ii) mieux budgétiser les coûts d'abreuvement qui pourraient être significatifs notamment lorsque l'eau provient du réseau public d'eau potable, iii) augmenter l'efficacité d'utilisation de l'eau en permettant une meilleure détection des fuites au niveau du 
réseau enterré qui peut être très important pour assurer l'abreuvement au pâturage. L'objectif de cette synthèse est de répertorier les outils disponibles dans la littérature pour quantifier les besoins en eau d'abreuvement des troupeaux laitiers. Il s'agira en particulier de rappeler les principes physiologiques qui régulent le bilan hydrique à l'échelle de l'animal, de donner une vue la plus exhaustive possible des équations de prédiction et des variables prédictives des besoins en eau propres aux vaches laitières et enfin de donner quelques éléments de réflexion pour prédire les besoins en eau d'abreuvement du troupeau.

\section{1 / Bilan hydrique de l'ani- mal et homéostasie}

\section{1 / Les compartiments et les flux d'eau dans l'animal}

Une vache contient de 55 à $70 \%$ d'eau selon son stade physiologique (Murphy 1992, Andrew et al 1995) et à un stade physiologique donné, une perte de $20 \%$ d'eau corporelle est fatale (NRC 2001). Les pertes d'eau corporelle sont indispensables à une multitude de fonctions vitales pour l'organisme comme i) la digestion et le métabolisme car l'eau est le diluant des excrétions urinaire et fécale, ii) la régulation de la température corporelle par la perte de chaleur latente cutanée et respiratoire lorsque la température ambiante augmente, iii) la production laitière car chaque $\mathrm{kg}$ de lait contient en moyenne $875 \mathrm{~g}$ d'eau (Alais 1984). Afin de permettre un renouvellement de l'eau corporelle, ces pertes d'eau doivent être compensées par des apports qui se composent de l'eau d'abreuvement, de l'eau contenue dans les aliments ingérés et, plus marginalement, de l'eau métabolique issue des réactions d'oxydation. L'ensemble des apports et des pertes d'eau chez la vache laitière constitue le bilan hydrique de l'animal (figure 1) et certains de ces flux sont étroitement régulés de façon à maintenir la quantité d'eau présente dans l'organisme.

L'eau corporelle englobe les compartiments hydriques intracellulaire et extracellulaire qui sont séparés par les membranes plasmatiques. Chez les ruminants, l'eau corporelle est contenue pour moitié dans le compartiment intracellulaire (Woodford et al 1984a, Murphy 1992). Cette proportion est nettement inférieure à celle de deux tiers généralement citée chez l'Homme (Essig et Friedlander 2009a), du fait du volume important des contenus digestifs des ruminants proportionnellement au volume corporel. A titre d'exemple, le volume ruminal d'une vache Holstein adulte est en moyenne de 100 à $150 \mathrm{~L}$ pour un poids vif de $600 \mathrm{~kg}$. Le compartiment d'eau extracellulaire comprend l'eau gastrointestinale, l'eau du plasma et les liquides interstitiels qui remplissent les espaces intercellulaires ou qui sont contenus dans les tissus conjonctifs (Murphy 1992). Chez les bovins, l'eau gastro-intestinale représente environ $30 \%$ de l'eau corporelle, cette proportion variant avec l'alimentation et les conditions environnementales (Andrew et al 1995). L'eau contenue dans le plasma et l'eau interstitielle ne représentent chacune qu'environ $10 \%$ de l'eau corporelle (Woodford et al 1984a).

\section{2 / Les forces motrices de la régulation des flux d'eau}

La fonction essentielle de l'eau dans l'organisme est d'assurer la solubilisation des molécules nécessaire à leurs interactions lors des réactions métabo- liques et à leurs mouvements dans les différents compartiments de l'organisme (Essig et Friedlander 2009a). Cette nécessité de solubilisation détermine le volume du compartiment hydrique extracellulaire ainsi que le volume des cellules ou volume hydrique intracellulaire. Le volume des cellules dépend avant tout des mouvements d'eau à travers la membrane plasmique entre les compartiments intra et extracellulaire, car celle-ci est plus perméable à l'eau qu'aux molécules dissoutes. Les mouvements d'eau sont déterminés par la tonicité des compartiments qui est définie par le nombre de molécules non diffusibles dissoutes par volume d'eau. Dans les cellules, les molécules non diffusibles sont essentiellement le potassium et le phosphate organique. Dans les liquides extracellulaires, le sodium est la principale substance non diffusible et la natrémie un indicateur de la tonicité de ce compartiment. Les différences de concentration sont maintenues notamment grâce à des pompes Na-K ATPase. Même si les cellules ont développé des mécanismes d'adaptation pour minimiser les variations du volume cellulaire, ceux-ci sont insuffisants pour faire face à la plupart des perturbations de tonicité subies par l'organisme. C'est donc à l'échelle de l'organisme que s'effectue l'adaptation des contenus en eau et en molécules dissoutes qui permet le maintien du volume cellulaire. Les perturbations initiales de tonicité, qu'elles soient liées à une altération du contenu en eau ou en molécules dissoutes, surviennent donc dans le compartiment extracellulaire et c'est à ce niveau que des régulations sont mises en œuvre avec comme consigne le maintien de la tonicité plasmatique et du volume sanguin.

Parmi les flux du bilan hydrique d'une vache laitière, ce sont l'eau bue et l'excrétion urinaire qui permettent

Figure 1. Les principaux flux d'eau à l'échelle d'une vache laitière nourrie avec une ration à base d'ensilage de maïs et en conditions de thermoneutralité (Khelil-Arfa 2012).

Dans cet essai la rétention d'eau corporelle était de $1 \mathrm{~L} / \mathrm{j}$.

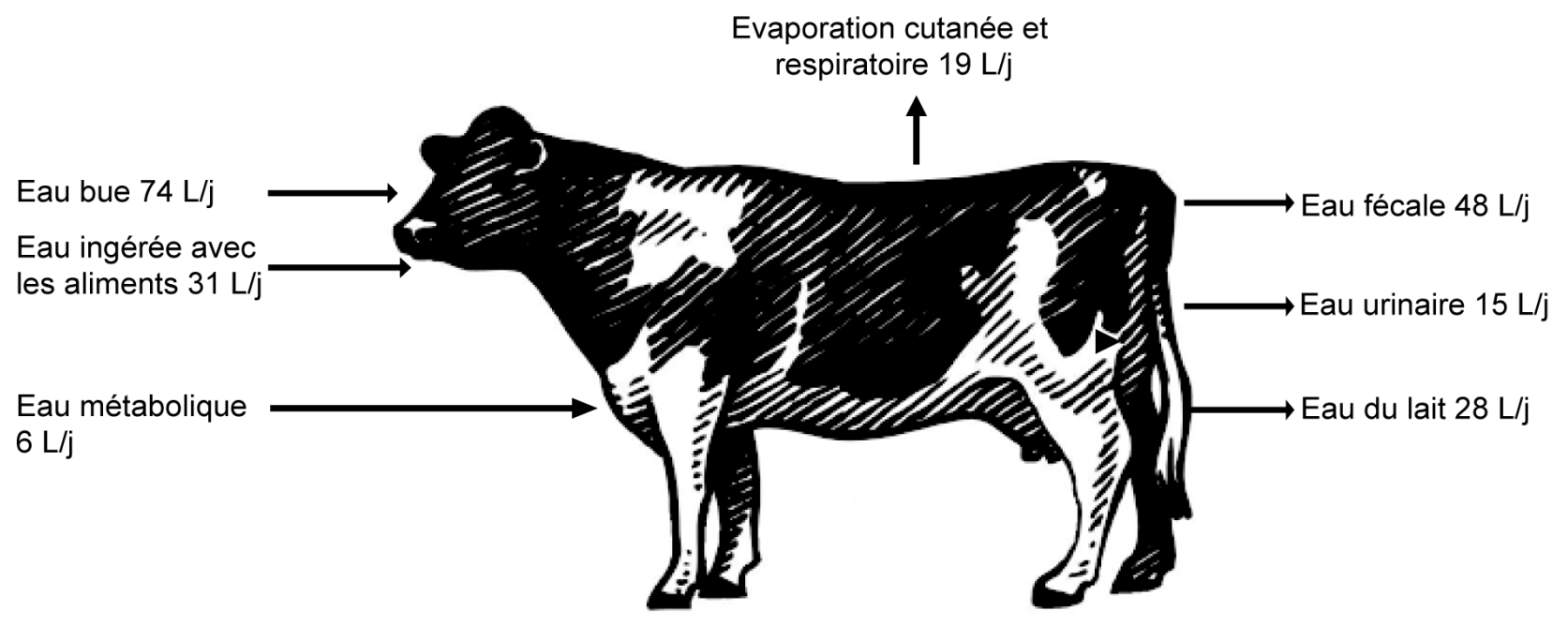


d'ajuster les entrées et les sorties d'eau à l'échelle de l'organisme pour réguler la pression osmotique et le volume sanguin. Les autres flux du bilan hydrique sont pour certains peu régulés, comme c'est le cas pour l'excrétion fécale, ou régulés par d'autres consignes que celle du maintien de l'eau corporelle, comme c'est le cas pour l'ingestion (régulée par les distensions du tube digestif ou le métabolisme énergétique) et pour l'évaporation cutanée et respiratoire (régulée de façon à maintenir la température corporelle) (Maia et al 2005a). Ces autres flux sont la cause des perturbations initiales de tonicité subies par l'organisme. En effet, les pertes digestives et sudorales ou respiratoires sont hypo-osmolaires par rapport au sang (Jenkinson et Mabon 1973) et entrâ̂nent donc une perte d'eau supérieure à celle des molécules dissoutes. De même, l'ingestion d'aliment s'accompagne de l'absorption par le tube digestif d'une quantité importante de molécules susceptibles de modifier la tonicité des compartiments hydriques.

\section{3 / Régulation de l'excrétion rénale}

Le rein a pour rôle principal de permettre l'élimination des substances hors de l'organisme telle que l'urée tout en maintenant la balance hydrique et électrolytique de l'organisme. Les néphrons qui en sont l'unité fonctionnelle, débutent par la capsule de Bowman qui encapsule une imbrication de capillaires au niveau du glomérule (Cunningham et Klein 2007). A ce niveau le sang est filtré, c'est-à-dire que les composants cellulaires et les protéines de poids moléculaires élevés à moyens sont conservés dans les capillaires alors que l'eau et les solutés passent dans le filtrat glomérulaire dont la composition est proche du plasma. Chez une vache laitière, le volume journalier filtré au niveau de la capsule de Bowman représente $2000 \mathrm{~L} / \mathrm{j}$ (KhelilArfa 2012). Pour concentrer l'urée, le filtrat glomérulaire va être soumis à une succession de concentrations et de dilutions lors de sa progression dans le néphron grâce à des mouvements d'absorption à travers les cellules tubulaires du néphron permis par des aquaporines et des transporteurs d'urée (Essig et Friedlander 2009a). Ainsi, le tube proximal du néphron qui prolonge le glomérule réabsorbe de façon iso-osmotique environ les deux tiers du filtrat glomérulaire. Environ $90 \%$ du filtrat sera réabsorbé en l'absence de régulation dans les premiers segments du néphron en amont du canal collecteur (Cunningham et Klein 2007). Ce n'est qu'au niveau des derniers segments du néphron (néphron distal) que les réabsorptions d'eau et de solutés sont régulées (Cunningham et Klein 2007).
Le volume d'eau excrété par voie urinaire dépend donc de la régulation au niveau rénal de la réabsorption d'eau, qui est avant-tout contrôlée par l'ADH ( Anti Diuretic Hormone »), aussi appelée vasopressine (Essig et Friedlander 2009a). L'ADH a pour effet d'augmenter la perméabilité à l'eau du tube collecteur, ce qui a pour effet d'augmenter l'osmolarité de l'urine excrétée et de réduire le volume excrété (Cunningham et Klein 2007). L'ADH est synthétisée dans l'hypothalamus et sécrétée par la posthypophyse de façon très rapide en réponse à une augmentation de la tonicité plasmatique et cette relation entre la sécrétion d'ADH et l'augmentation de la tonicité plasmatique est amplifiée en situation d'hypovolémie, c'est-à-dire de déficit de volume sanguin dans le système circulatoire (Essig et Friedlander 2009a). L'ADH est également stimulée par l'angiotensine II au sein du système rénine-angiotensine-aldostérone (Cunningham et Klein 2007). Ce système est stimulé par la baisse du volume sanguin et de la natrémie pour maintenir la balance hydro-sodée de l'organisme.

\section{4 / Les mécanismes de la soif}

Nous avons vu qu'un déficit en eau intra et extracellulaire entraîne une baisse de l'excrétion urinaire, mais ces mécanismes de régulation ne peuvent restaurer le volume hydrique corporel initial à long terme sans une activation conjointe de la soif (McKinley et Johnson 2004, Essig et Friedlander 2009a). Les stimuli de déclenchement de la soif sont similaires à ceux de la réabsorption rénale. Un déficit de volume plasmatique de plus de $8-10 \%$ au niveau cardiaque ou dans les vaisseaux sanguins où des barorécepteurs sont présents provoque une soif significative (Fitzsimons 1998) et un déficit de 1 à $2 \%$ d'eau cellulaire, ou de pression osmotique du milieu extracellulaire déclenche la soif (Fitzsimons 1998). Ces stimuli activent plus ou moins directement une zone cérébrale souvent appelée centre de la soif (Fitzsimons 1998). Cette zone permet l'intégration par le système nerveux central des signaux hormonaux, neuronaux et chimiques périphériques susceptibles de déclencher la soif (McKinley et Johnson 2004). Elle a aussi un rôle central sur l'appétit en sodium et les régulations cardio-vasculaires (Fitzsimons 1998) et est fortement stimulée par l'angiotensine II au sein du système rénine-angiotensinealdostérone (Fitzsimons 1998, McKinley et Johnson 2004, Essig et Friedlander 2009a). Cette zone est enfin sous la dépendance d'afférences oropharyngées inhibitrices, stimulées par l'ingestion d'eau, permettant d'arrêter l'apport hydrique avant que ce dernier ne provoque une chute brutale de tonicité plasmatique (Essig et Friedlander 2009a).

\section{2 / La quantification des besoins en eau des vaches laitières : équations de pré- diction et principaux fac- teurs de variation}

\section{1 / Les équations de prédiction de l'eau bue et de l'eau totale ingérée}

De nombreuses équations ont été établies pour prédire les quantités d'eau bue ou d'eau totale ingérée par les vaches laitières (tableau 1), l'eau totale ingérée étant la somme de l'eau bue et de l'eau contenue dans les aliments ingérés. Toutes ces équations ont été établies par régression multiple pour la majeure partie, ou par régression simple. Le nombre de données qui sous-tend ces équations est très variable, de plus de 2000 données journalières déclarées (Meyer et al 2004, Cardot et al 2008), à moins de 30 données qui sont parfois des moyennes sur plusieurs jours et plusieurs individus (Murphy et al 1983, Stockdale et King 1983, Dahlborn et al 1998, Kume et al 2010). Un autre élément qui différencie les jeux de données supportant de ces équations est la gamme de variabilité des rations, des niveaux de production et des conditions météorologiques. Ainsi les équations de Stockdale et King (1983) ont été exclusivement développées à partir de données de vaches laitières au pâturage, celles de Kume et al (2010) à partir de données de vaches nourries avec des rations à base d'ensilage d'herbe et celle de Murphy et al (1983) à partir de données de vaches nourries avec une même ration composée de $40 \%$ d'ensilage de maïs et $60 \%$ de concentré avec différents niveaux de complémentation sodée. Le tableau 2 résume les conditions d'obtention des équations recensées dans la littérature.

\section{2 / Les principaux facteurs de variation de l'eau bue et de l'eau totale ingérée chez la vache lai- tière}

a) La teneur en matière sèche de la ration

Il ressort clairement des équations répertoriées dans le tableau 1 que la première variable prédictive des quantités d'eau bue par les vaches laitières est la teneur en Matière Sèche (MS) de la ration. Cette variable apparaît dans 11 des 14 équations répertoriées. Deux des trois équations n'incluant pas la teneur en MS de la ration avaient été établies sur des données dans lesquelles la ration distribuée était toujours identique (Little et Shaw 1978, Murphy et al 1983). L'équation de Meyer et al (2004) est la 
Tableau 1. Les équations de prédiction recensées de l'eau bue et de l'eau totale ingérée journalières par les vaches laitières.

\begin{tabular}{|c|c|c|c|c|c|}
\hline Sources & Lieu & $\mathbf{N}^{(1)}$ & $R^{2(1)}$ & Pred $^{(1)}$ & Equations eau bue/totale ingérée (L/j) ${ }^{(2)}$ \\
\hline Castle et Thomas (1975) & Royaume-Uni & 66 & $3^{\mathrm{ETR}}$ & EB & {$[1]=2,53 \times P L+0,45 \times M S \%-15,30$} \\
\hline Little et Shaw (1978) & Royaume-Uni & 112 & 0,58 & EB & {$[2]=2,15 \times \mathrm{MSI}+0,73 \times \mathrm{PL}+12,3$} \\
\hline Stockdale et King (1983) & Australie & 15 & 0,96 & EB & {$[3]=-9,37+2,3 \times \mathrm{MSI}+0,53 \times \mathrm{MS} \%$} \\
\hline Murphy et al (1983) & $\begin{array}{l}\text { Urbana, } \\
\text { Etats-Unis }\end{array}$ & 19 & 0,59 & EB & {$[4]=15,99+1,58 \times \mathrm{MSI}+0,90 \times \mathrm{PL}+0,05 \times \mathrm{Nal}+1,20 \times \mathrm{Tmin}$} \\
\hline $\begin{array}{l}\text { Holter et Urban (1992) } \\
\text { (vaches en lactation) }\end{array}$ & $\begin{array}{l}\text { Durham } \\
\text { Etats-Unis }\end{array}$ & 329 & $\begin{array}{l}0,69 \\
0,19\end{array}$ & EB & $\begin{array}{l}{[5]-32,39+2,47 \times \mathrm{MSI}+0,6007 \times \mathrm{PL}+0,62 \times \mathrm{MS} \%+0,091 \times \mathrm{JD}} \\
-0,000257 \times \mathrm{JD}^{2} \\
{[6]=-68,28+4,76 \times \mathrm{MS} \%-0,038 \times \mathrm{MS}^{2} \%}\end{array}$ \\
\hline $\begin{array}{l}\text { Holter et Urban (1992) } \\
\text { (vaches taries) }\end{array}$ & $\begin{array}{l}\text { Durham } \\
\text { Etats-Unis }\end{array}$ & 60 & $\begin{array}{l}0,46 \\
0,42\end{array}$ & EB & {$[7]=-10,34+0,22 \times \mathrm{MS} \%+2,21 \times \mathrm{MSI}+0,039 \times \mathrm{MATr}^{2} \%$} \\
\hline Dahlborn et al (1998) & Suède & 24 & 0,67 & EB & {$[8]=14,3+1,24 \times \mathrm{PL}+0,32 \times \mathrm{MS} \%$} \\
\hline Meyer et al (2004) & Allemagne & 12821 & 0,60 & EB & {$[9]=-26,12+1,516 \times$ Tmoy $+1,29 \times \mathrm{PL}+0,058 \times \mathrm{PV}+0,40 \times \mathrm{Nal}$} \\
\hline Cardot et al (2008) & France & 1837 & 0,45 & EB & $\begin{array}{l}{[10]=1,54 \times M S I+1,33 \times P L+0,89 \times M S \%+0,57 \times \operatorname{Tmin}-0,3} \\
x \text { Pluv }-26,65\end{array}$ \\
\hline $\begin{array}{l}\text { Kume et al (2010) } \\
\text { (vaches en lactation) }\end{array}$ & Japon & 16 & 0,66 & EB & {$[11]=1,58 \times \mathrm{MS} \%-3,4$} \\
\hline $\begin{array}{l}\text { Kume et al (2010) } \\
\text { (vaches taries) }\end{array}$ & Japon & 30 & 0,67 & EB & {$[12]=0,91 \times M S \%-18,8$} \\
\hline Kheli-Arfa et al (2012) & France & 232 & $\begin{array}{l}0,92 \\
0,86\end{array}$ & EB & $\begin{array}{l}{[13]=0,83 \times \mathrm{MS} \%+3,22 \times \mathrm{MSI}+0,92 \times \mathrm{PL}-0,28 \times \mathrm{CONC} \%} \\
+0,037 \times \mathrm{PV}-77,6 \\
{[14]=0,97 \times \mathrm{MS} \%+1,54 \times \mathrm{PL}-0,29 \times \mathrm{CONC} \%+0,039 \times \mathrm{PV}-41,1}\end{array}$ \\
\hline $\begin{array}{l}\text { Paquay et al (1970a) } \\
\text { (vaches taries) }\end{array}$ & Belgique & 32 & $\begin{array}{l}0,88 \\
0,93\end{array}$ & ETI & $\begin{array}{l}{[15]=5,47 \times \mathrm{MSI}-2,58 \text { (aliment humide) }} \\
{[16]=4,55 \times \mathrm{MSI}-7,31 \text { (aliment sec) }}\end{array}$ \\
\hline Stockdale et King (1983) & Australie & 8 & 0,99 & ETI & {$[17]=11,34+4,63 \times \mathrm{MSI}-0,0036 \mathrm{MS} \%+0,84$ Tmoy } \\
\hline $\begin{array}{l}\text { Holter et Urban (1992) } \\
\text { (vaches taries) }\end{array}$ & $\begin{array}{l}\text { Durham } \\
\text { Etats-Unis }\end{array}$ & 60 & 0,99 & ETI & $\begin{array}{l}{[18]=35,19+0,98 \times \mathrm{EB}-0,011 \times \mathrm{PV}+1,081 \times \mathrm{MSI}+1,18 \times \mathrm{MATr} \%} \\
-0,038 \times \mathrm{MATr}^{2} \%-0,99 \times \mathrm{MS} \%+0,0054 \times \mathrm{MS} \%^{2}\end{array}$ \\
\hline $\begin{array}{l}\text { Kume et al (2010) } \\
\text { (vaches en lactation) }\end{array}$ & Japon & 16 & $\begin{array}{l}0,56 \\
0,26 \\
0,86 \\
0,50 \\
0,85\end{array}$ & ETI & $\begin{array}{l}{[19]=36,3 \times \mathrm{K} \text { ration } \%+27,4} \\
{[20]=4,15 \times \mathrm{MATr} \%+29,4} \\
{[21]=6,24 \times \mathrm{MSI}-31,0} \\
{[22]=0,129 \times \mathrm{Nal}+26,4} \\
{[23]=0,14 \times \mathrm{KI}+40,6}\end{array}$ \\
\hline $\begin{array}{l}\text { Kume et al (2010) } \\
\text { (vaches taries) }\end{array}$ & Japon & 30 & $\begin{array}{l}0,53 \\
0,36 \\
0,40 \\
0,63 \\
0,74\end{array}$ & ETI & $\begin{array}{l}{[24]=7,43 \times \mathrm{K} \text { ration } \%+12,8} \\
{[25]=1,51 \times \mathrm{MATr} \%+7,60} \\
{[26]=5,91 \times \mathrm{MSI}-15,2} \\
{[27]=0,14 \times \mathrm{Nal}+4,28} \\
{[28]=0,10 \times \mathrm{KI}+11,1}\end{array}$ \\
\hline Khelil-Arfa et al (2012) & France & 232 & $\begin{array}{l}0,87 \\
0,82\end{array}$ & ETI & $\begin{array}{l}{[29]=3,89 \times \mathrm{MSI}+9,4 \cdot 10^{-3} \times \mathrm{MATf}+0,81 \times \mathrm{PL}-0,8 \times \mathrm{MATC}-0,94} \\
{[30]=1,56 \times \mathrm{PL}+1,9 \times \mathrm{MATf}+43,3}\end{array}$ \\
\hline
\end{tabular}

${ }^{(1)} \mathrm{N}$ : Nombre de données ; $\mathrm{R}^{2}$ de l'équation donnée dans la publication ; Pred : variable prédite, EB : Eau Bue (L/j), ETI : Eau Totale Ingérée (L/j) ; ETR : Ecart-Type Résiduel.

${ }^{(2)} \mathrm{MSI}$ : Matière Sèche Ingérée $(\mathrm{kg} / \mathrm{j})$; MS \% : Teneur en Matière Sèche de la ration $(\%)$; PL : Production Laitière $(\mathrm{kg} / \mathrm{j})$;

$\mathrm{PV}$ : Poids Vif $(\mathrm{kg})$; Tmin : Température ambiante minimale journalière $\left({ }^{\circ} \mathrm{C}\right)$; Tmoy : Température ambiante moyenne $\left({ }^{\circ} \mathrm{C}\right)$;

Pluv : Pluviométrie journalière $(\mathrm{mm} / \mathrm{j})$; JD : Rang du jour julien dans l'année en cours ; MATr: Teneur en Matière Azotée

Totale de la ration (\% MS) ; MATf : Teneur en Matière Azotée de la ration provenant du fourrage (\% MS) ; MATc : Teneur en

Matière Azotée de la ration provenant du concentré (\% MS) ; Nal : Quantités journalières de sodium ingéré ( $\mathrm{g} / \mathrm{j})$;

$\mathrm{KI}$ : Potassium ingéré $(\mathrm{g} / \mathrm{j})$. 
Tableau 2. Gammes de variation des jeux de données utilisés pour construire les équations de prédiction des besoins en eau des vaches laitières (moyenne \pm écart-type).

\begin{tabular}{|c|c|c|c|c|c|c|c|c|c|c|}
\hline Auteurs & Nb. & $\begin{array}{c}\mathbf{N}^{\circ} \\
\text { Equat. }\end{array}$ & MS $(\%)^{(1)}$ & $\underset{(\mathbf{k g} / \mathbf{j}){ }^{(2)}}{\text { MSI }}$ & $\underset{(k g / j)}{P L}$ & $\begin{array}{c}\mathrm{PV} \\
(\mathbf{k g})^{(4)}\end{array}$ & $\begin{array}{l}\text { MAT } \\
(\%)^{(5)}\end{array}$ & $\begin{array}{c}\mathrm{Na} \\
(\mathrm{g} / \mathrm{kg})\end{array}$ & $\begin{array}{c}\mathrm{K} \\
(\mathrm{g} / \mathrm{kg})\end{array}$ & $\begin{array}{l}\text { Temp } \\
\left({ }^{\circ} \mathrm{C}\right)\end{array}$ \\
\hline (a) & 1752 & 15,16 & $\begin{array}{l}\text { Ensilages, } \\
\text { herbe verte } \\
\text { et foin }\end{array}$ & $7,6 \pm 2,11$ & 0 & $550 \pm 75$ & & & & \\
\hline (b) & 66 & 1 & $50 \pm 22,4$ & $15,3 \pm 2,19$ & $16.8 \pm 2,84$ & & & & & $8,2 \pm 2.57$ \\
\hline (c) & 112 & 2 & $87 \pm 1.5$ & $13,3 \pm 0,14$ & $21,4 \pm 0,31$ & $476 \pm 12,2$ & $15.0 \pm 0.27$ & $1,33 \pm 0,024$ & $10,3 \pm 0,19$ & $15,0 \pm 0,72$ \\
\hline (d) & 15 & 3 & $30 \pm 12,2$ & $10.2 \pm 2.74$ & & & & & & $16,7 \pm 3,2$ \\
\hline (e) & 8 & 17 & $46 \pm 28$ & $13,2 \pm 2.42$ & & & & & & $13,2 \pm 2,4$ \\
\hline (f) & 76 & 4 & $62 \pm 5^{(6)}$ & $19,0 \pm 3,99$ & $33,1 \pm 6,13$ & $580 \pm 30$ & $17,0 \pm 0,34$ & $4.0 \pm 2,33$ & & $13,6 \pm 6,92$ \\
\hline (g) & 329 & 5,6 & $50 \pm 7,2$ & $18,7 \pm 2,8$ & $34,6 \pm 6,8$ & $640 \pm 49$ & $16,1 \pm 2,1$ & & & \\
\hline (h) & 60 & 7 & $60 \pm 24,4$ & $10,3 \pm 2,27$ & 0 & $640 \pm 49$ & $16 \pm 3,2$ & & & \\
\hline (i) & 24 & 8 & $70 \pm 21,8$ & $18,2 \pm 1.9$ & $25 \pm 2,6$ & $624 \pm 5,7^{(7)}$ & & & & \\
\hline (j) & 12821 & 9 & $55 \pm 9,5$ & $20,5 \pm 3,9$ & $31,1 \pm 7,7$ & $628 \pm 61$ & & $2,1 \pm 0,25$ & $17,0 \pm 1,80$ & $8,6 \pm 7,1$ \\
\hline (k) & 1837 & 10 & $48 \pm 5,0$ & $20,6 \pm 3,3$ & $26,5 \pm 5,9$ & & $14,5 \pm 0,7$ & & & $3.8 \pm 4,4^{(8)}$ \\
\hline (I) & 16 & $\begin{array}{l}11,19, \\
20,21, \\
22,23\end{array}$ & $51 \pm 9,5$ & $20,7 \pm 2,2$ & $29,5 \pm 3,5$ & $609 \pm 48$ & $16,6 \pm 2.0$ & & $19,6 \pm 3,2$ & $20 \pm 2,0$ \\
\hline (m) & 30 & $\begin{array}{l}12,24, \\
25,26, \\
27,28\end{array}$ & $38 \pm 11,0$ & $7,7 \pm 1,0$ & 0 & $619 \pm 66$ & $15 \pm 3,8$ & & $23,5 \pm 9,3$ & $20 \pm 2,0$ \\
\hline (n) & 232 & $\begin{array}{l}13,14 \\
29,30\end{array}$ & $61 \pm 29,1$ & $17.8 \pm 4,11$ & $24.9 \pm 8,36$ & $630 \pm 86,6$ & & & & $15 \pm 2,0$ \\
\hline
\end{tabular}

(1) MS : Matière Sèche ; ${ }^{(2)} \mathrm{MSI}$ : Matière Sèche Ingérée ; ${ }^{(3)}$ : PL : Production Laitière ; ${ }^{(4)} \mathrm{PV}$ : Production Viande : ${ }^{(5)}$ MAT : Matière Azotée Totale ; ${ }^{(6)}$ écart-type estimé, ${ }^{(7)}$ moyenne de 2 données sur 2 lots en début d'essai, ${ }^{(8)}$ min $0,1 \pm 4$.

(a) Paquay et al (1970a) Vaches taries ; (b) Castle et Thomas (1975) ; (c) Little et Shaw (1978) ; (d) Stockdale et King (1983) Eq. [3] ; (e) Stockdale et King (1983) Eq. [17] ; (f) Murphy et al (1983) ; (g) Holter et Urban (1992) Vaches en lactation ; (h) Holter et Urban (1992) Vaches taries ; (i) Dahlborn et al (1998) ; (j) Meyer et al (2004); (k) Cardot et al (2008) ; (I) Kume et al (2010) Vaches en lactation; (m) Kume et al (2010) Vaches taries (n) Khelil-Arfa et al (2012).

seule équation à ne pas faire ressortir la teneur en MS de la ration comme paramètre prédictif malgré une forte variabilité de ce paramètre dans le jeu de données, peut-être du fait d'une forte variabilité associée des quantités de sodium ingéré, des productions laitières ou des températures ambiantes.

A contrario, la teneur en MS de la ration a beaucoup moins d'influence sur les quantités d'eau totale ingérée (tableau 1, Khelil-Arfa et al 2012). La teneur en MS de la ration est la réciproque de la teneur en eau de la ration. Dans le tube digestif, l'eau bue et l'eau contenue dans les aliments ingérés ont des devenirs légèrement différents (Woodford et al 1984b, Faichney et Boston 1985, Cafe et Poppi 1994), mais elles contri- buent toutes deux à la dilution du milieu intérieur et à la mise en œuvre des régulations hydriques à l'échelle de l'organisme. L'eau contenue dans les aliments est rarement suffisante pour permettre à l'animal de réguler la tonicité et le volume plasmatique et l'ingestion d'eau couvre les besoins en eau non couverts par la ration. Cela a été vérifié par de nombreuses observations expérimentales. Ainsi, lorsque la teneur en MS de la ration est le principal facteur de variation, lors de séchage ou de trempage partiel de la ration par exemple, il ressort très clairement que les quantités d'eau bue par les vaches laitières diminuent linéairement lorsque le taux de MS diminue, jusqu'à devenir quasiment nulles lorsque ce dernier atteint 11 à $13 \%$, alors que les quantités d'eau totale ingérée ne varient proportionnellement pas (CabreraEstrada 2003). Ainsi avec des rations à base de fourrages conservés secs, l'eau bue représente jusqu'à $78-90 \%$ de la quantité totale d'eau entrant dans le bilan hydrique des vaches laitières (Holter et Urban 1992, Cardot et al 2008) alors que cette proportion peut être inférieure à $30 \%$ avec des rations humides à base d'ensilage ou au pâturage (Stockdale et King 1983).

\section{b) L'ingestion d'aliment et la produc-} tion laitière

Si la teneur en MS de la ration ressort comme la première variable prédictive des quantités d'eau bue par les vaches laitières, c'est la quantité de MS ingérée qui ressort comme la première variable 
prédictive de l'eau totale ingérée dans la majorité des équations recensées (Paquay et al 1970a et b, Stockdale et King 1983, Kume et al 2010, Khelil-Arfa et al 2012). C'est aussi le premier facteur de variation de la quantité d'eau bue dans les équations établies sur des jeux de données avec très peu de variabilité de la teneur en MS de la ration (Little et Shaw 1978, Murphy et al 1983, Stockdale et King 1983) et le second dans les équations établies sur des jeux de données avec une forte variabilité de la teneur en MS de la ration (Holter et Urban 1992, Khelil-Arfa et al 2012). Avec les jeux de données utilisés pour établir les équations de prédiction des quantités d'eau bue ou d'eau totale ingérée de KhelilArfa et al (2012), le retrait de la MS ingérée des variables prédictives candidates a réduit considérablement la précision des équations. Dans quelques équations prédictives des quantités d'eau bue, la quantité de MS ingérée apparaît comme un prédicteur moins bon que la production laitière (Castle et Thomas 1975, Dahlborn et al 1998, Meyer et al 2004). Cependant, chez les vaches laitières, la quantité de MS ingérée est très corrélée à la production laitière, d'autant plus si les données utilisées incluent des observations uniquement de vaches en lactation, avec peu de variabilité du potentiel génétique ou du stade de lactation. Cette corrélation peut être à l'origine de colinéarité, ce qui explique sans doute la grande variabilité des pentes de l'un et de l'autre de ces facteurs dans les régressions multiples. A titre d'exemple, la pente de la quantité d'eau bue va de 1,54 à $2,47 \mathrm{~L} / \mathrm{kg}$ de MS ingérée et de 0,60 à $2,53 \mathrm{~L} / \mathrm{kg}$ lait sur les équations répertoriées dans le tableau 1.

En absence de charge osmotique élevée à l'échelle de l'organisme et de stress thermique, il a été établi chez plusieurs espèces de mammifères que la quantité d'eau bue est proportionnelle à la consommation énergétique. Chez l'Homme, les apports journaliers recommandés ont été fixés dans de nombreux pays entre 1 à $1,5 \mathrm{~mL} / \mathrm{kcal}$ d'énergie brute ingérée (EFSA 2010). Chez les ruminants, Silanikove (1989) a montré avec des chèvres taries de différentes races adaptées ou non à la chaleur, que la quantité d'eau totale ingérée était reliée à la quantité d'énergie ingérée à raison de $1,1 \mathrm{~mL} / \mathrm{kcal}$ d'énergie brute ingérée ou $0,9 \mathrm{~mL} / \mathrm{kcal}$ d'énergie digestible. Cette relation entre l'ingestion d'énergie et d'eau serait due à la proportionnalité entre la consommation d'oxygène et le taux de renouvellement du compartiment hydrique corporel, proportionnalité qui serait très stable lorsque l'on compare plusieurs genres et espèces de mammifères (Macfarlane et Howard 1972, Silanikove 1989). Cependant, cette relation entre l'ingestion d'eau et d'énergie ne résulte pas d'un système de régulation commun ou de relations stœchiométriques identifiées impliquant ces deux entités, mais plutôt de quelques points de convergence entre les régulations du métabolisme énergétique et celles de l'équilibre hydrique. Le point de convergence le plus important est que l'acte d'ingestion d'aliment en soi entraine une séquence d'ingestion d'eau, de façon très systématique chez les monogastriques, mais aussi dans une moindre mesure chez les ruminants (Langhans et al 1995). La causalité entre l'ingestion d'aliment et l'ingestion d'eau s'explique notamment par des stimuli sensoriels lors de l'ingestion d'aliment, comme une sensation de sécheresse dans la bouche et la mise en œuvre de la sécrétion salivaire, mais aussi par l'occurrence de stimuli osmotiques au niveau du tractus intestinal suite à l'arrivée d'aliment, ou encore par une hypertonicité du milieu intérieur suite à l'absorption des nutriments, qui n'est observée que suite à l'ingestion de gros repas après un jeûne, mais qui est surement anticipée par l'animal après apprentissage (Langhans et al 1995).

Chez les ruminants, en l'absence de stress thermique, les recommandations d'apports en eau ont été rarement exprimées en fonction de l'énergie ingérée. Les recommandations de l'INRA (1988), reprises par l'ANSES (2010), donnent un besoin en eau de l'ordre de 4,5 à 5,5 L/kg de Matière Sèche Ingérée (MSI) pour des vaches laitières. Ce besoin correspond environ aux pentes des équations [15] et [16] de Paquay et al (1970a) (tableau 1) obtenues pour des vaches taries alimentées avec des rations humides à base d'ensilage ou d'herbe (pente de 4,5 L/kg MS ingérée), ou avec des rations sèches à base de foin (pente de 5,5 L/kg MS ingérée). Il est possible que ces recommandations soient un peu faibles pour des vaches en lactation si l'eau contenue dans le lait n'est pas prise en compte dans les quantités recommandées d'eau totale ingérée, en considérant par exemple une teneur en eau moyenne du lait de $87,5 \%$ (Alais 1984). Si on considère grossièrement que l'aliment est une ration équilibrée avec une teneur moyenne de $1 \mathrm{UFL} / \mathrm{kg} \mathrm{MS}$, soit $1700 \mathrm{kcal} / \mathrm{kg} \mathrm{MS}$, le chiffre de $5 \mathrm{~L}$ d'eau/kg MS ingérée conduit à une estimation de 2,4 mL/kcal d'EB ingérée ce qui est élevé par rapport à la valeur de $1 \mathrm{~mL} / \mathrm{kcal} \mathrm{d}$ 'EB ingérée établie par Silanikove (1989). Il est probable que ces écarts s'expliquent en partie par des différences de teneurs des rations en électrolytes ou en matières azotées qui doivent être excrétés par voie urinaire.

c) Effets des teneurs en sodium, potassium et azote de la ration

Chez des vaches laitières non soumises à des situations de stress thermique, les pertes urinaires d'eau sont en premier lieu déterminées par les teneurs en électrolytes, et notamment en sodium et en potassium, ainsi qu'en azote de la ration (Bannink et al 1999). Il est vraisemblable que les pertes urinaires d'eau soient en partie corrélées aux besoins en eau des vaches. Ainsi, les teneurs en électrolytes et en azote de la ration devraient faire varier de façon notable les besoins en eau pour un même niveau d'ingestion. L'EFSA (2010) définit pour l'Homme une charge rénale potentielle en électrolytes qui est calculée à partir de la somme des moles de $\mathrm{Na}, \mathrm{K}, \mathrm{Cl}$, P et $\mathrm{N}$ ingérées. Les teneurs en électrolytes ou en azote de la ration ont été considérées de façons très hétérogènes dans les équations de prédiction recensées, notamment du fait de la difficulté de les quantifier précisément.

Deux équations du tableau 1 proposent d'intégrer la teneur en sodium de la ration dans les variables prédictives des quantités d'eau bue (Murphy et al 1983, Meyer et al 2004). L'équation de Murphy et al (1983) résulte de mesures dans des essais visant à quantifier l'effet d'ajouts de sels minéraux dans l'alimentation de vaches laitières et elle a donc été établie sur un jeu de données présentant une grande variabilité de la teneur en sodium des rations. La relation positive entre les volumes d'eau bue et l'ingestion de sodium est due au fait que le sodium est le principal indicateur de la tonicité du volume hydrique corporel extracellulaire (cf. $\S$ 1.2). Ainsi, l'augmentation de la teneur en sodium plasmatique en réponse à l'augmentation d'ingestion de sodium déclenche directement la soif du fait de la hausse de la tonicité plasmatique associée (Essig et Friedlander 2009b). De plus, le bilan sodique de l'organisme est avant tout régulé au niveau du rein, notamment par le système rénine-angiotensine-aldostérone, par l'intermédiaire duquel une hausse de l'osmolarité plasmatique a pour effet d'augmenter conjointement l'excrétion urinaire de sodium et d'eau. Dans la pratique, il est très difficile d'estimer les quantités de sodium ingéré par les vaches laitières. Les aliments pour ruminants ont des teneurs souvent trop faibles pour couvrir les besoins physiologiques d'animaux productifs (Meschy 2010) et il est alors nécessaire de laisser à disposition du troupeau des pierres à sel dont l'ingestion peut être très variable d'un jour à l'autre ou entre individus, et difficilement mesurable. C'est une des raisons qui fait que ce paramètre est peu présent dans les équations de prédiction.

Parmi les équations recencées dans le tableau 1, Kume et al (2010) sont les seuls à avoir identifié un effet de la quantité de potassium ingérée sur l'ingestion d'eau. Ceci est cohérent avec le 
fait que le potassium ingéré est aussi une variable prédictive du volume urinaire excrété chez le ruminant (Bannink et al 1999). A la différence du sodium, les rations alimentaires des ruminants sont souvent excédentaires en potassium, notamment si elles sont basées sur du pâturage ou sur de l'ensilage d'herbe (Meschy 2010). Les liaisons entre les mécanismes de régulation de l'excrétion $\mathrm{du}$ potassium et du bilan hydrique sont moins claires que pour le sodium. Comme le sodium, le potassium est principalement excrété au niveau du rein, du moins en cas d'absence d'insuffisance rénale avérée (Houillier 2009). La régulation de l'excrétion du potassium est avant tout mise en œuvre par une augmentation de la sécrétion d'aldostérone suite à un excès durable de potassium dans l'organisme (Houillier 2009). L'aldostérone a pour effet de favoriser la sécrétion de potassium au niveau du tube collecteur des néphrons, ce qui pourrait s'accompagner d'une moindre réabsorption d'eau du fait du gradient d'osmolarité croissant entre la lumière du tube collecteur et les zones cortico-papillaires interstitielles (Houillier 2009).

La teneur en Matière Azotée Totale (MAT) de la ration est une variable prédictive dans une des équations de prédiction de l'eau bue recensées dans le tableau 1 (Holter et Urban 1992) et dans quelques équations de prédiction de l'eau totale ingérée (Kume et al 2010 , Khelil-Arfa et al 2012). L'équation de prédiction de l'eau totale ingérée de Khelil-Arfa et al (2012) inclut même distinctement la teneur en MAT provenant du fourrage de la ration et la teneur en MAT provenant du concentré de la ration. Un des rôles du rein est de permettre l'excrétion rénale d'urée qui est aussi un élément important de l'osmolarité de l'urine (Bannink et al 1999). Ainsi, une ingestion importante d'azote par rapport aux besoins de l'animal entraine aussi une hausse de la teneur en urée plasmatique, une excrétion accrue d'urée urinaire (Burgos et al 2007) et une augmentation du volume urinaire (Bannink et al 1999).

Enfin, il est possible que la teneur en MS de la ration puisse constituer un indicateur indirect, mais assez simple à caractériser de la charge osmotique de la ration. Ceci pourrait expliquer qu'elle apparaisse comme une variable prédictive secondaire de l'eau totale ingérée dans plusieurs équations (Paquay et al 1970a, Stockdale et King 1983, Holter et Urban 1992). La teneur en MS de la ration est positivement corrélée à la teneur en MAT et en potassium des rations lorsqu'on que l'on compare des rations ou des fourrages (Paquay et al 1970a, Khelil-Arfa et al 2012). Ceci est sans doute encore plus vrai pour les plantes entières pour lesquelles les teneurs en protéines sont liées à l'importance des contenus cellulaires, ce qui peut expliquer la relation avec les électrolytes et l'existence d'une meilleure relation pour les fourrages que pour les aliments concentrés.

\section{d) Effets des paramètres météorologiques}

Pour des vaches laitières en lactation, il est établi que les pertes d'eau par évaporation cutanée et respiratoire augmentent de façon exponentielle avec la température ambiante et deviennent significatives dès que la température dépasse $14^{\circ} \mathrm{C}$ (Silanikove 2000, Maia et al 2005a et b, figure 2). Ces pertes ne sont pas toujours perceptibles à l'œil nu car elles comportent des pertes d'eau par voie respiratoire ainsi que des pertes d'eau par voie cutanée qui peuvent se produire sans transpiration apparente lorsque la température reste modérée. On parle alors de perspiration insensible. Malgré tout, ces pertes peuvent avoir des répercussions très importantes sur le bilan hydrique de l'animal et elles doivent être compensées par les mécanismes cités dans la première partie pour maintenir tonicité et volémie sanguine. Les pertes d'eau par évaporation permettent aux vaches laitières de réguler leur température corporelle autour de $38^{\circ} \mathrm{C}$ en particulier dans des conditions de fortes températures selon les mécanismes de régulation thermique des animaux homéothermes notamment décrits dans la revue de Silanikove (2000). Chaque litre d'eau évaporée permet la dissipation de $2500 \mathrm{~kJ}$.

Pourtant, seule une minorité des équations de prédiction des quantités d'eau bue ou d'eau totale ingérée recensées dans le tableau 1 inclut des paramètres prédictifs se rapportant aux conditions météorologiques et en particulier à la température ambiante. Lorsqu'ils sont inclus, ces paramètres sont soit la température ambiante minimale ou moyenne, associée ou non à la pluviométrie (Murphy et al 1983, Stockdale et King 1983, Meyer et al 2004, Cardot et al 2008) ou des critères indirects comme le rang de jour julien dans l'année en cours (Holter et Urban 1992). La raison de la faible prise en compte des conditions météorologiques est d'une part, qu'elles n'ont sans doute pas toujours été caractérisées dans les jeux de données qui sous-tendent les équations du tableau 1 et, d'autre part, que la température ambiante est restée assez faible et peu

Figure 2. Effet de la température moyenne sur la quantité d'eau évaporée par une vache laitière en lactation de 600 kg d'après le modèle de Maia et al (2005a et b) et sur le surplus d'eau nécessaire à l'évaporation d'après l'équation simplifiée de Boudon et al (2012).

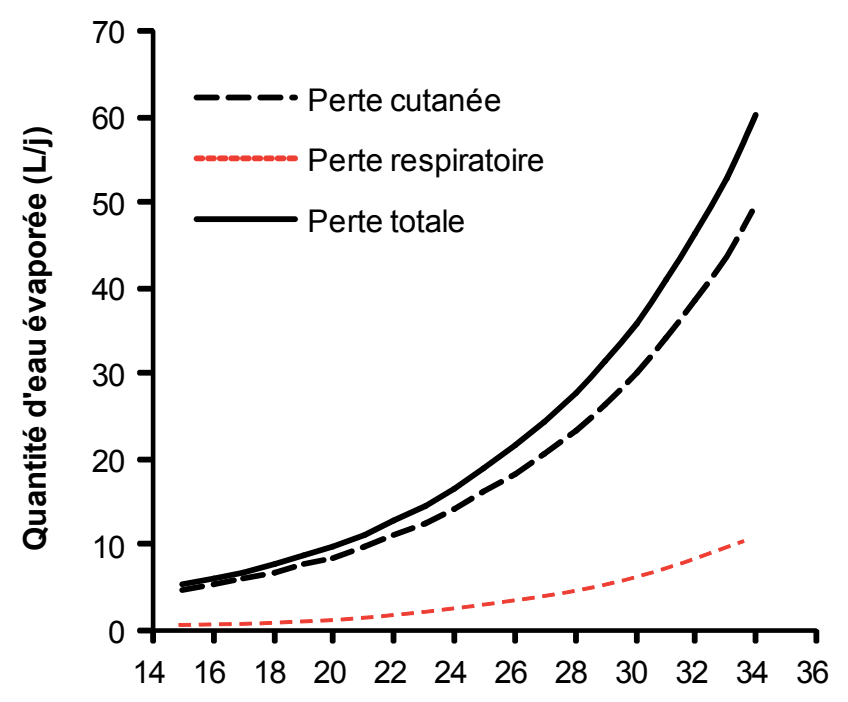

Température moyenne journalière $\left({ }^{\circ} \mathrm{C}\right)$

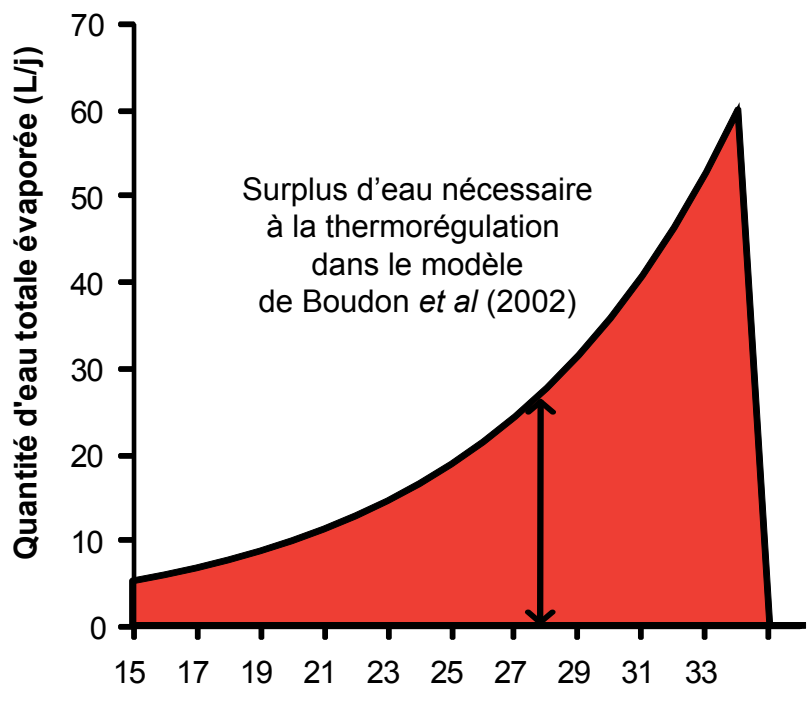

Température moyenne journalière $\left({ }^{\circ} \mathrm{C}\right)$ 
variable lorsqu'elle a été mesurée (tableau 2). De plus, l'approche par régression multiple qui a été privilégiée pour l'établissement des équations du tableau 1 , peut entraîner des confusions partielles entre des paramètres météorologiques et d'autres paramètres liés à la conduite des animaux comme le stade de lactation ou les conditions d'alimentation très variables avec la saison. Ceci peut nuire fortement à l'extrapolation d'équations établies dans un système et sur un site géographique donné à d'autres contextes.

Certains travaux, non répertoriés dans le tableau 1 proposent également des correctifs de l'effet de la température sur les besoins en eau des vaches laitières, à ajouter aux besoins calculés par ailleurs en conditions de température modérée. Un correctif a été intégré aux équations de prédictions de Khelil-Arfa et al (2012) des besoins en eau dès que la température ambiante dépasse $15^{\circ} \mathrm{C}$. Ce correctif, présenté à la figure 2 , prend en compte l'effet de la température journalière moyenne sur la quantité d'eau évaporée par des vaches en lactation, estimé par les équations de Maia et al (2005a et b) obtenues à partir des mesures directes de l'évaporation par des capsules cutanées et des masques respiratoires. La validité de cette démarche repose sur l'observation que lorsque la température ambiante augmente, l'augmentation des quantités d'eau évaporée par les vaches laitières est entièrement compensée par une augmentation des quantités d'eau bue lorsque ces flux sont rapportés à la MS ingérée (Khelil-Arfa 2012). Il a été vérifié par les travaux de Khelil-Arfa et al (2012) que les modèles de Maia et al (2005a et b) qui ont l'avantage de la simplicité, pouvaient s'adapter pour des vaches laitières dans d'autres contextes de mesure. Par ailleurs, il a aussi été proposé par l'INRA (1988) de majorer les besoins en eau de 30, 50 ou $100 \%$ si la température ambiante, vraisemblablement maximale journalière, dépassait respectivement 20,25 ou $30^{\circ} \mathrm{C}$.

Au-delà de la température ambiante, la pluviométrie apparaît comme une variable prédictive des quantités d'eau bue par les vaches laitières dans l'équation de Cardot et al (2008), mais ce paramètre, bien que significatif dans la régression n'explique qu'une très faible proportion, inférieure à $0,1 \%$, de la variabilité de l'eau bue. Les mécanismes sous-jacents ne sont pas clairement connus. Il est établi que l'humidité relative joue un rôle important sur l'adaptation à la chaleur car elle peut compromettre les capacités de l'animal à dissiper la chaleur corporelle et conduire à des baisses notables d'ingestion de MS (Silanikove 2000). Cependant, l'équation de Cardot et al (2008) a été établie dans des conditions de température ambiante plutôt faible. De plus, même en situation de forte chaleur, on peut supposer que l'effet potentiel de l'humidité relative sur l'ingestion d'eau puisse en grande partie être pris en compte à travers l'ingestion des animaux qui est une variable prédictive clé de l'ingestion d'eau. En effet, il est vraisemblable que la pluviométrie et la hausse d'humidité relative qui en découlent puissent être partiellement confondues avec la teneur en MS de la ration. Au pâturage, les travaux de Cabrera-Estrada (2003) ont bien montré que la pluviométrie peut avoir un effet très important sur la teneur en MS de l'herbe et que cela avait des répercussions négatives sur l'ingestion d'eau.

\section{3 / Évaluation de la précision des équations de prédiction des quantités d'eau bue}

a) Démarche

Les équations de prédiction des besoins en eau des vaches laitières recensées dans le tableau 1 ont été établies dans des contextes divers de rations,

Tableau 3. Caractéristiques du jeu de données utilisé pour évaluer les équations : moyennes, écarts-types, minimum et maximum des valeurs pour les principales variables de production, de ration et d'eau ingérée (89 données $\left.{ }^{(1)}\right)$.

\begin{tabular}{|c|c|c|c|c|}
\hline & Moyenne & $\mathrm{ET}^{(2)}$ & Min & Max \\
\hline \multicolumn{5}{|l|}{ Variable de Production } \\
\hline Quantité ingérée (kg/j) & 15,7 & 4,91 & 5,3 & 27,1 \\
\hline Production de lait $(\mathrm{kg} / \mathrm{j})$ & 21,5 & 11,88 & 0,0 & 41,5 \\
\hline Poids vif (kg) & 585 & 96,40 & 358 & 786 \\
\hline \multicolumn{5}{|l|}{ Composition des rations } \\
\hline Matière sèche $(\mathrm{g} / 100 \mathrm{~g})$ & 54,8 & 15,22 & 37,9 & 86,0 \\
\hline Proportion de concentré (g/100 g) & 45,1 & 19,55 & 0,0 & 70,0 \\
\hline $\mathrm{MAT}^{(3)}(\mathrm{g} / 100 \mathrm{~g} \mathrm{MS})$ & 14,4 & 3,71 & 3,5 & 19,8 \\
\hline Température ambiante ${ }^{\circ} \mathrm{C}$ & 21,3 & 7,82 & 5,0 & 36,0 \\
\hline \multicolumn{5}{|l|}{ Flux d'eau (L/j) } \\
\hline Eau bue & 66,5 & 27,52 & 10,9 & 128,0 \\
\hline Eau dans l'aliment & 15,9 & 8,78 & 0,8 & 36,9 \\
\hline Eau totale ingérée & 82,6 & 31,04 & 26,8 & 144,5 \\
\hline
\end{tabular}

(1) Références des études utilisées : Chaiyabutr et al 2008 (Int. J. Biometeorol., 52, 575-585), Dado et Allen 1994 (J. Dairy Sci., 77, 132-144), Dewhurst et al 1998, (Anim. Sci., 66, 543-550), Doelman et al 2008 (J. Anim. Sci., 91, 3998-4001), Escobosa et al 1984, (J. Dairy Sci., 67, 574-584), Khelil-Arfa, 2012, Gustafson et al 2000 (Acta Agric. Scand., Sect.A, Animal Sci., 50, 111-120), Knowlton et al 2010, (J. Dairy Sci., 93, 407-412), Kojima et al 2005, (Anim. Sci., 76, 139-145), Kurihara et al 1984 (Jpn. J. Livest. Manage., 20, 61-67), Mc Dowell et al 1968 (J. Dairy Sci., 52, 188-194), Osborne et al 2002 (Can. J. Anim. Sci., 267-272), Osborne et al 2009 (J. Anim. Sci., 92 , 698-707), Richards 1985 (Trop. Anim. Health Prod., 17, 209-217), Shalit et al 1991 (J. Dairy Sci., 74, 1874-1883), Silanikove et al 1997 (J. Dairy Sci., 80, 945-956), Solomon et al 1995 (J. Dairy Sci., 78, 620-624), Steiger Burgos et al 2001 (Am. J. Physiol. Regul. Integr. Comp. Physiol., 280, 418-527), Thomas et al 2007 (J. Anim. Sci., 90, 3831-3837).

(2) ET : Ecart-Type.

${ }^{(3)}$ MAT : Matière Azotée Totale. 
Encadré. La décomposition de l'erreur de prédiction (Bibby et Toutenburg 1977).

Le carré de l'erreur moyenne de prédiction $\left(\mathrm{EMP}^{2}\right)$ se définit de la façon suivante : $E M P^{2}=\frac{1}{n} \sum_{i=1}^{n}(P i=O i)^{2}$

où $\mathrm{n}$ est le nombre d'observation, $\mathrm{Pi}$ la valeur prédite et Oi la valeur observée pour l'observation i.

La décomposition algébrique de l'EMP conduit à la formule suivante :

$E M P^{2}=(\bar{P}=\bar{O})^{2}=\left(s_{P}=r s_{O}\right)^{2}=\left(1=r^{2}\right) s_{O}^{2}$

$E M P^{2}=$ biais sur la moyenne + biais sur la pente + dispersion des données

où $\bar{P}$ et $\bar{O}$ sont les moyennes des prédits et des observés, $s_{p}$ et $s_{o}$ sont les écarts-types des prédits et des observés et $r$ est le coefficient de corrélation entre les prédits et les observés.

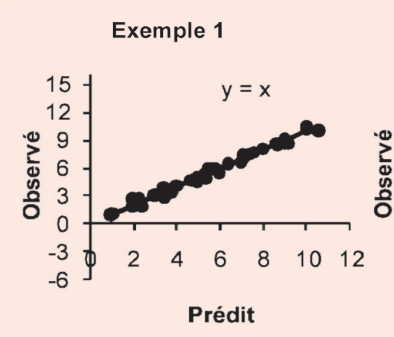

$\mathrm{EMP}=0,35$

Biais sur la moyenne $=2,9 \%$

Biais sur la pente $=0,0 \%$

Dispersion $=97,1 \%$
Exemple 2

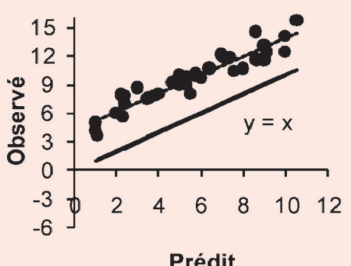

$\mathrm{EMP}=4,12$

Biais sur la moyenne $=95,3 \%$

Biais sur la pente $=0,2 \%$

Dispersion $=4,5 \%$

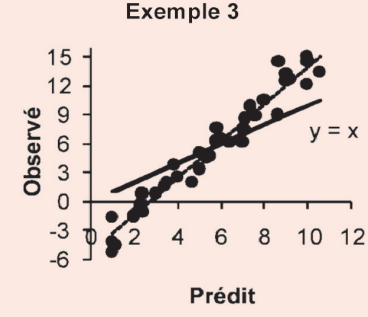

$\mathrm{EMP}=2,90$

Biais sur la moyenne $=0,8 \%$ Biais sur la pente $=95,0 \%$ Dispersion $=4,2 \%$ de productions laitières ou de conditions météorologiques et climatiques et il nous a semblé intéressant d'évaluer leur erreur moyenne de prédiction sur un jeu de données indépendant de toutes les équations et issu de contextes géographiques variés. Le jeu de données utilisé a été celui construit par Khelil-Arfa et al (2012) pour réaliser une validation externe des équations de prédiction des besoins en eau des vaches laitières que ces auteurs ont établi à la thermoneutralité, soit pour des températures ambiantes inférieures à $20^{\circ} \mathrm{C}$. Quelques modifications ont été apportées à ce jeu de données depuis la publication de KhelilArfa et al (2012) : i) toutes les données qui avaient pu avoir été utilisées pour établir une quelconque équation de prédiction des flux d'eau comparée ont été exclues, ii) 18 données expérimentales de Khelil-Arfa et al (2012) n'ayant pas servi à l'établissement des équations [13], [14], [29] et [30] ont été incluses. Le jeu de données final inclut 89 mesures d'eau bue sur des lots de vaches laitières soumises à un même traitement expérimental dans 20 études réalisées entre 1969 et 2010. Les références des études incluses dans le jeu de données ainsi que les gammes de variation des niveaux d'ingestion et de production, de composition de la ration, de température ambiante et d'eau bue et ingérée sont données dans le tableau 3.

Les équations dont nous avons comparé les erreurs de prédiction étaient les équations [1], [2], [3], [4], [8], [9], [10],
[17] (tableau 1), les systèmes d'équations [5] et [7], [11] et [12] selon que les vaches étaient en lactation ou pas, [13] et [31] (figure 2) selon que la température ambiante était supérieure ou non à $15^{\circ} \mathrm{C}$ et enfin [15] et [16] selon que la ration était à base de foin ou d'herbe et d'ensilage. Dans le cas des équations [15], [16] et [17], la variable prédite était la quantité d'eau totale ingérée et non l'eau bue. Les quantités d'eau ingérée avec l'aliment ont donc été soustraites des quantités prédites afin de disposer d'une prédiction de l'eau bue. Nous avons également inclus les recommandations INRA (1988) dans cette comparaison. Certaines variables prédictives n'ont pas pu être renseignées de façon précise pour certaines observations du jeu de données. Pour les équations de Murphy et al (1983) et de Meyer et al (2004), la quantité de sodium ingérée était rarement disponible dans les publications qui ont servi à établir le jeu de données et nous avons fait l'hypothèse que les quantités de sodium ingéré correspondaient aux besoins des animaux calculés selon les recommandations du NRC (2001). La température ambiante était disponible pour 63 observations et supérieure à $15^{\circ} \mathrm{C}$ pour 54 observations. Quand la température n'était pas disponible, nous l'avons estimée égale à une température de thermoneutralité de $15^{\circ} \mathrm{C}$ après avoir vérifié la saison et la localisation géographique de l'essai. Dans les équations de Cardot et al (2008) et Murphy et al (1983), nous avons considéré que la température minimale était équivalente à la température ambiante moyenne journalière moins $5^{\circ} \mathrm{C}$. Dans les équations de Holter et Urban (1992), le rang du jour julien dans l'année n'a pas été renseigné bien que ce paramètre soit le moyen de prendre en compte l'effet des conditions météorologiques sur l'ingestion d'eau.

Les Erreurs Moyennes de Prédiction (EMP) des équations ont été calculées comme la racine de la moyenne des carrés des différences entre les valeurs prédites et observées. L'EMP relative a été calculée comme le rapport de l'EMP sur la valeur moyenne observée d'eau bue. Le carré de l'EMP a été décomposé en trois sources d'erreur selon Bibby et Toutenburg (1977), liées à un biais sur la moyenne, à un biais sur la pente et à la dispersion entre les données observées et prédites (cf. encadré).

\section{b) Des performances prédictives contras-} tées

L'erreur de prédiction moyenne est de $25,6 \mathrm{~L} / \mathrm{j}$ ce qui représente $36 \%$ de la moyenne de l'eau bue observée, ce qui est un chiffre élevé par rapport aux erreurs de prédiction rapportées dans les publications. Cependant, le tableau 4 et la figure 3 illustrent une variabilité importante dans les performances de prédiction des équations sur ce jeu de données. L'erreur de prédiction la plus faible est de $15,4 \mathrm{~L} / \mathrm{j}$, soit $23,2 \%$ de la moyenne de l'eau bue observée pour l'équation de Boudon et al (2012) et la plus élevée de $31,6 \mathrm{~L} / \mathrm{j}$ (Kume et al 2010), si on exclut l'erreur de prédiction des recommandations INRA (1988) dont l'objectif était de recommander un apport d'eau permettant un abreuvement ad libitum des animaux avec une certaine marge de sécurité.

Pour cinq des équations testées, la proportion de l'EMP attribuable à un biais sur la moyenne est supérieure à $50 \%$ (Murphy et al 1983, INRA 1988, Holter et Urban 1992, Meyer et al 2004, Cardot et al 2008). Cependant pour la majorité de ces équations, le biais provient avant tout des estimations qui ont dû être réalisées dans le jeu de données pour renseigner toutes les variables prédictives. Ceci illustre bien la difficulté à renseigner certaines variables et la nécessité de considérer la liste des variables prédictives dont on dispose pour choisir une équation. La température minimale est, dans deux équations, considérée comme indicateur des conditions météorologiques (Murphy et al 1983, Cardot et al 2008) alors que la température moyenne est plus souvent donnée dans les publications. Les essais de Khelil-Arfa (2012) ont montré que lorsque l'on comparait des dynamiques journalières de température ambiante, la température moyenne 
Figure 3. Relation entre les quantités d'eau bue mesurées et celles prédites par les équations de prédiction de l'eau bue existantes. - Eau bue obtenue à des températures $<15^{\circ} \mathrm{C}$. $\circ$ Eau bue obtenue à des températures $>15^{\circ} \mathrm{C}$.

La droite en noir représente la bissectrice $y=x$.
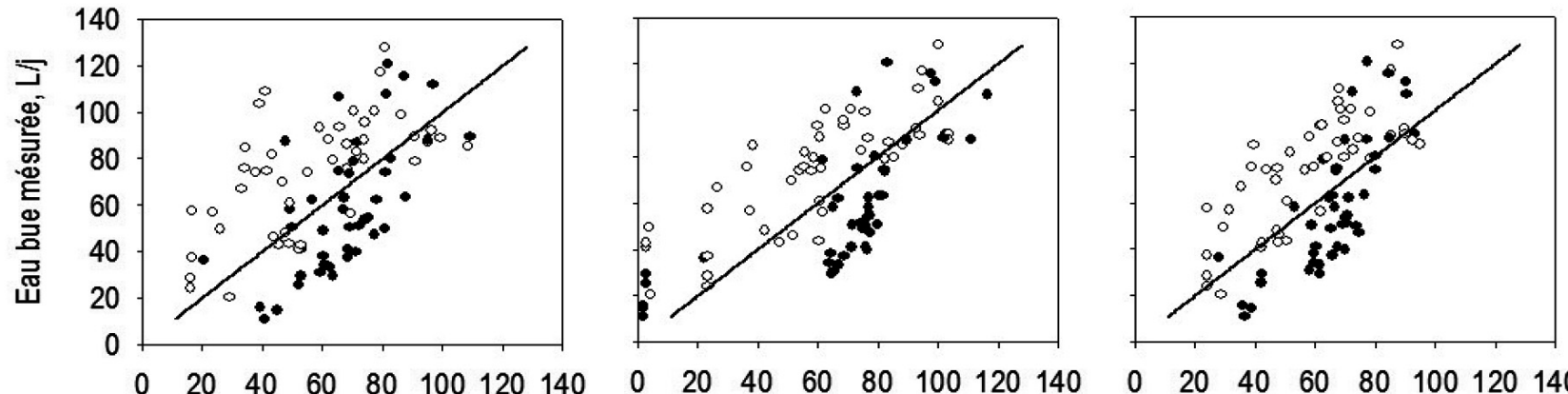

Eau ingérée Paquay et al (1970a) [15,16] Eau bue Castle et Thomas (1975) [1]
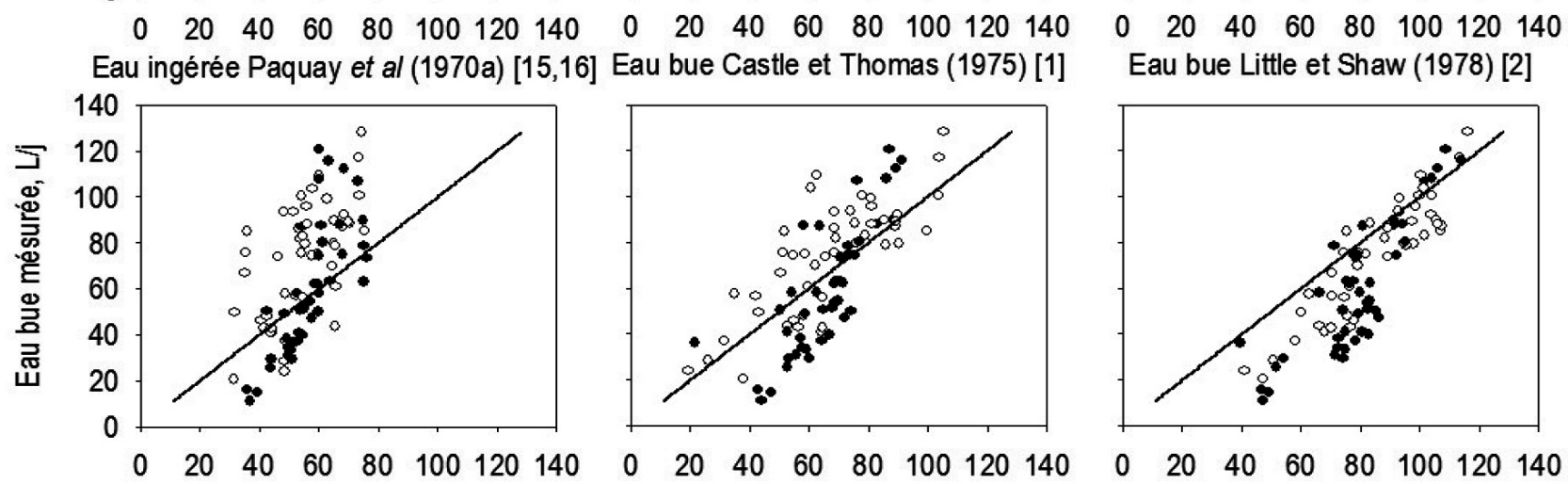

Eau bue Little et Shaw (1978) [2]

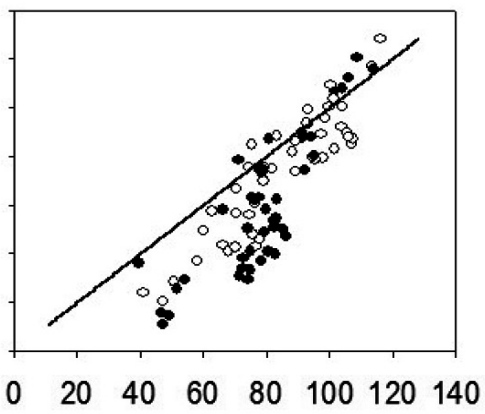

Eau bue Stockdale et King (1983) [3] Eau ingérée Stockdale et King (1983) [17] Eau bue Murphy et al (1983) [4]
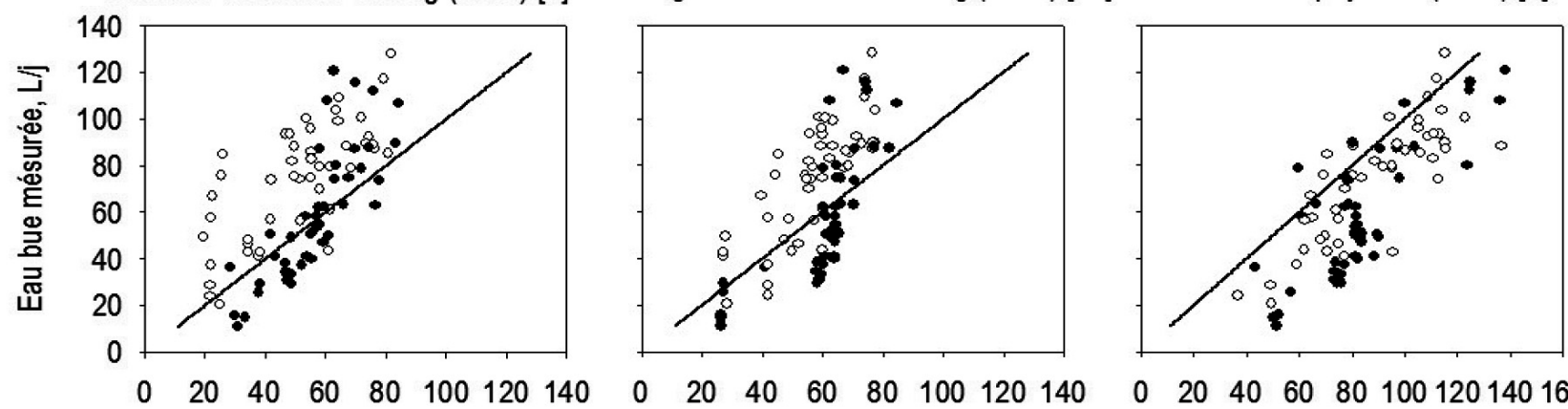

Eau bue Holter et Urban (1992) [5, 7]

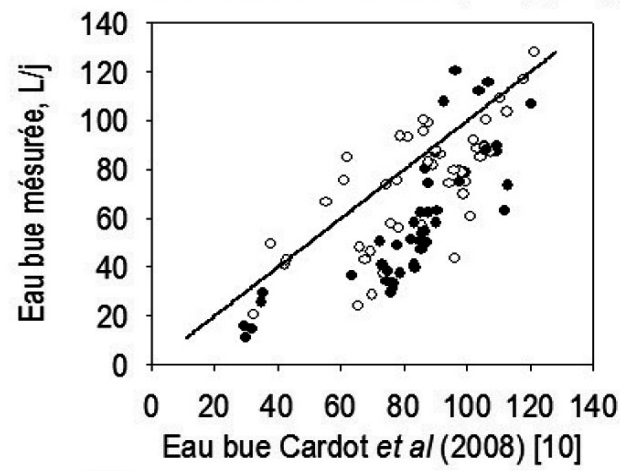

Eau bue Dahlborn et al (1998) [8]

$\begin{array}{llllll}0 & 20 & 40 & 60 & 80 & 100120140160\end{array}$ Eau bue Meyer et al (2004) [9]
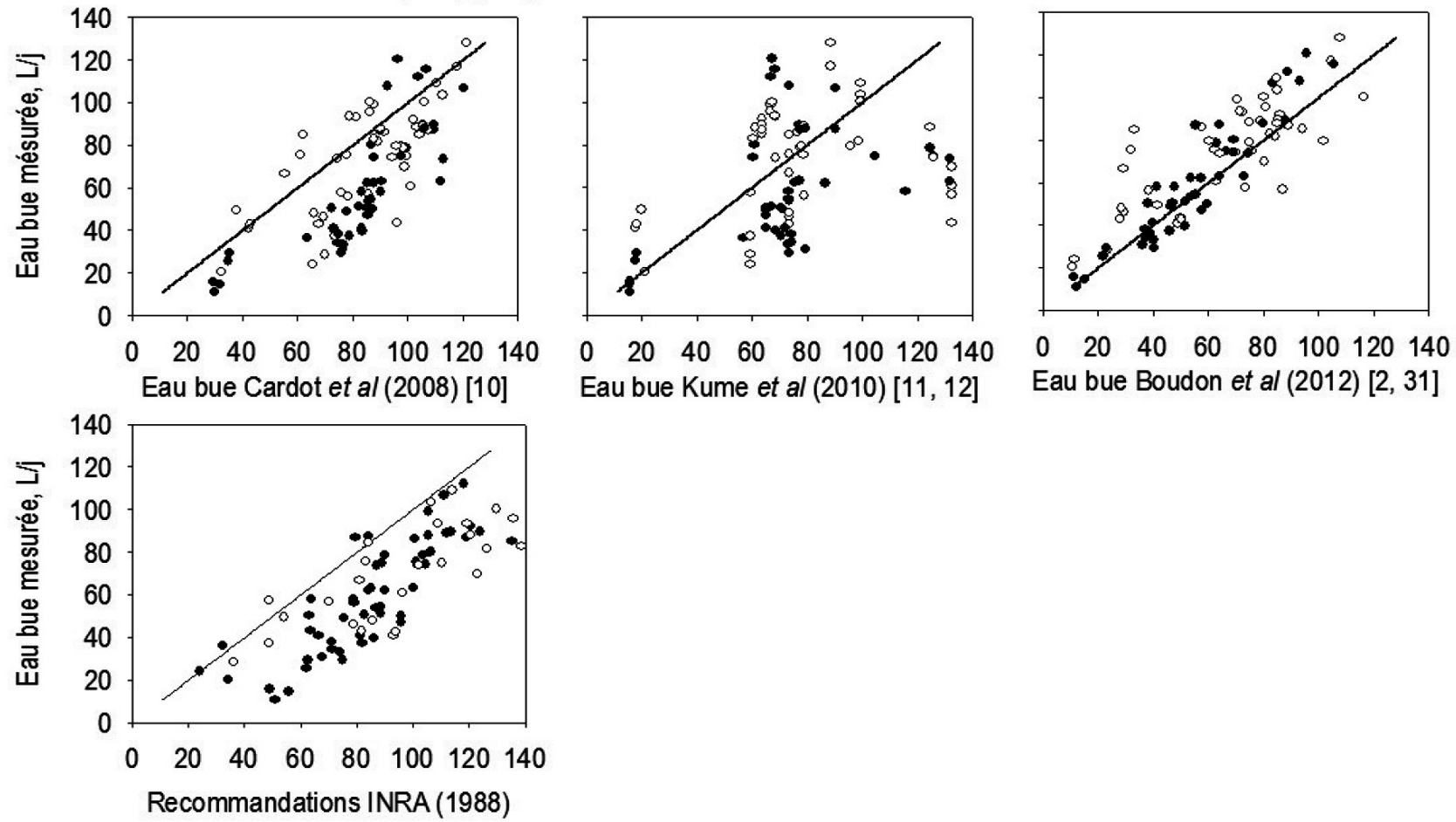
Tableau 4. Erreurs Moyennes de Prédiction (EMP) de l'eau bue et décomposition de ces erreurs pour les principales équations de prédictions publiées appliquées au jeu de 89 données dont 39 à la thermoneutralité.

\begin{tabular}{|c|c|c|c|c|c|c|c|}
\hline \multirow[b]{2}{*}{ Equations } & \multirow[b]{2}{*}{ EMP (L/j) } & \multirow{2}{*}{$\begin{array}{c}\text { EMP relative } \\
(\%)\end{array}$} & \multicolumn{3}{|c|}{ Décomp. $(\%)^{(1)}$} & \multirow{2}{*}{$\begin{array}{c}\text { EMP } \\
\operatorname{TN}(L / j)^{(2)}\end{array}$} & \multirow{2}{*}{$\begin{array}{l}\text { EMP relative } \\
\text { TN }(\%)^{(2)}\end{array}$} \\
\hline & & & $\begin{array}{l}\text { Biais } \\
\text { moy. }\end{array}$ & $\begin{array}{l}\text { Biais } \\
\text { Pente }\end{array}$ & Dispers. & & \\
\hline $\begin{array}{l}\text { Paquay et al (1970a), } \\
\text { éq. } 15,16^{(3)}\end{array}$ & 25,24 & 38,0 & 3,47 & 9,15 & 87,38 & 22,45 & 34,5 \\
\hline $\begin{array}{l}\text { Castle et Thomas (1975), } \\
\text { éq. } 1\end{array}$ & 22,82 & 34,3 & 1,61 & 20,11 & 78,28 & 22,31 & 33,4 \\
\hline $\begin{array}{l}\text { Little et Shaw (1978), } \\
\text { éq. } 2\end{array}$ & 21,39 & 32,2 & 5,22 & 0,05 & 94,73 & 18,24 & 28,4 \\
\hline $\begin{array}{l}\text { Stockdale et King (1983), } \\
\text { éq. } 3\end{array}$ & 24,78 & 37,3 & 19,21 & 5,70 & 75,09 & 15,39 & 27,6 \\
\hline $\begin{array}{l}\text { Stockdale et King (1983), } \\
\text { éq. } 17^{(3)}\end{array}$ & 18,54 & 27,9 & 0,05 & 2,41 & 97,54 & 19,38 & 30,7 \\
\hline $\begin{array}{l}\text { Murphy et al (1983), } \\
\text { éq. } 4\end{array}$ & 21,47 & 32,8 & 50,89 & 8,79 & 40,32 & 27,42 & 36,2 \\
\hline $\begin{array}{l}\text { Holter et Urban (1992), } \\
\text { éq. } 5,7\end{array}$ & 24,22 & 36,4 & 28,52 & 0,45 & 71,03 & 12,43 & 22,5 \\
\hline $\begin{array}{l}\text { Dahlborn et al (1998), } \\
\text { éq. } 8\end{array}$ & 22,15 & 33,3 & 13,31 & 4,08 & 82,61 & 15,32 & 25,7 \\
\hline $\begin{array}{l}\text { Meyer et al (2004), } \\
\text { éq. } 9\end{array}$ & 25,48 & 38,3 & 58,07 & 0,01 & 41,92 & 30,85 & 39,4 \\
\hline $\begin{array}{l}\text { Cardot et al (2008), } \\
\text { éq. } 10\end{array}$ & 25,03 & 37,6 & 46,84 & 0,21 & 52,94 & 30,30 & 37,5 \\
\hline $\begin{array}{l}\text { Kume et al (2010), } \\
\text { éq. } 11 \text { et } 12\end{array}$ & 31,63 & 47,6 & 6,78 & 29,75 & 63,48 & 28,82 & 40,2 \\
\hline $\begin{array}{l}\text { Boudon et al (2012), } \\
\text { éq. } 2 \text { et } 31\end{array}$ & 15,40 & 23,2 & 17,36 & 0,62 & 82,03 & 10,29 & 20,5 \\
\hline INRA (1988) & 34,75 & 52,3 & 68,52 & 10,18 & 21,30 & 31,33 & 38,8 \\
\hline
\end{tabular}

${ }^{(1)}$ Décomp. : Décomposition de l'erreur selon Bibby et Toutenburg (1977); (2) EMP et EMP relative sur les 39 données obtenues à la thermoneutralité, c'est-à-dire pour une température ambiante moyenne inférieure ou égale à $15^{\circ} \mathrm{C}$;

(3) Calcul de l'eau bue en soustrayant l'eau ingérée avec l'aliment à l'eau totale ingérée.

était une meilleure variable prédictive des pertes d'eau corporelle par évaporation à l'échelle de la journée. Cette température moyenne peut être estimée sur le terrain comme la moyenne des températures minimale et maximale journalières. Les recommandations d'apport en eau pour les vaches laitières de l'INRA (1988) ont systématiquement été supérieures au volume d'eau bue mesuré. Le biais que nous avons observé sur l'équation de Holter et Urban (1992) provient avant tout du fait que nous n'avons pas renseigné le rang de jour julien dans l'équation. Ceci est très bien illustré par le fait que cette équation a donné de très bonnes performances de prédiction de l'eau bue lorsque les données étaient restreintes à des conditions de thermoneutralité.

La variabilité des données qui ont servi à établir les équations doit aussi être un facteur clé dans le choix des équations de prédiction des besoins en eau. Les équations de Castle et Thomas (1975) et de Kume et al (2010) se caractérisent par une proportion d'erreur sur la pente élevée par rapport aux autres équations, ce qui est sans doute lié à la faible variabilité à la fois des niveaux de production et des conditions météorologiques dans ces études. De même, certaines équations sont avant tout adaptées à des conditions de thermoneutralité, comme celles de Holter et Urban (1992) si le rang de jour julien n'est pas renseigné, ou celles de Stockdale et King (1983) et de Dahlborn et al (1998) dont les erreurs de prédictions sont bien moindres sur les données obtenues dans des conditions de thermoneutralité.

\section{3 / Passer de l'estimation des besoins de la vache laitière à ceux du troupeau laitier}

Les équations prédictives des besoins en eau des vaches laitières décrites cidessus ne sont pas suffisantes pour calculer avec le moins de biais possible les besoins en eau du troupeau laitier pour deux raisons principales que nous développons ci-dessous.

\section{1 / Un biais systématique entre les mesures d'eau bue individuel- les et en troupeau}

Les données qui sous-tendent les équations de prédiction des besoins en eau d'abreuvement publiées et recen- sées dans le tableau 1 ont été obtenues avec des vaches dont l'ingestion d'eau était mesurée de façon individuelle. Même si cela n'a pas été systématiquement précisé dans les publications, il est très probable que l'abreuvement était assuré par des abreuvoirs de type « bols à tube ou à palette ». Boudon et al (2012) ont observé que leur modèle de prédiction des besoins en eau d'abreuvement des vaches laitières sous-estimait d'environ $13 \%$ les quantités d'eau consommée par un lot de 60 vaches laitières abreuvées en commun sur des abreuvoirs à niveau constant et dont la consommation d'eau ainsi que l'alimentation et la production ont été suivies sur une année (figure 4). Les raisons de cet écart de prédiction peuvent être multiples mais un effet probable du type d'abreuvoirs serait très cohérent avec les résultats de Pinheiro Machado Filho et al (2004) qui montrent que des vaches laitières peuvent consommer 30 à $50 \%$ d'eau en plus lorsque la surface de l'abreuvoir est doublée. Cette publication est à notre connaissance la seule à avoir quantifié les conséquences sur les volumes d'eau consommée, de la préférence largement constatée dans la littérature des vaches laitières pour les abreuvoirs à niveau constant par rapport aux abreuvoirs de type « bols 
Figure 4. Comparaison entre les données d'eau bue prédites par les équations [13] (Khelil-Arfa et al 2012) et [31] (Boudon et al 2012) et les données collectées sur un lot de 60 vaches laitières suivies pendant un an à la ferme expérimentale des Trinottières (Maine et Loire).

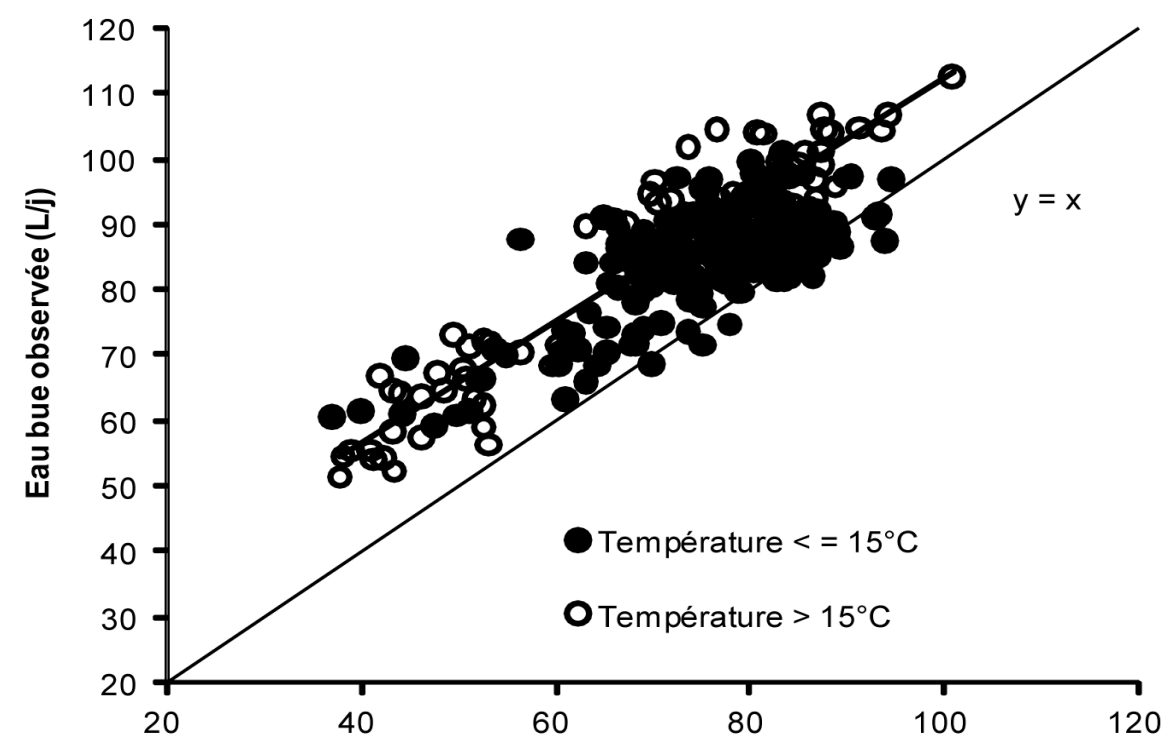

Eau bue prédite par l'équation [13] en incluantle correctif de l'effet tem pérature de l'équation [31](L/j)

à tube ou à palette " (Teixeira et al 2006, Artaux et al 2011). Les raisons de cette plus grande consommation, gaspillage lié aux éclaboussures ou augmentation de la quantité d'eau réellement bue, sont difficiles à établir et n'ont pas été précisément quantifiées à ce jour.

\section{2 / Les besoins en eau des génisses}

La prise en compte des besoins totaux des troupeaux laitiers nécessite aussi de comptabiliser les besoins en eau d'abreuvement des génisses pour lesquelles
Ménard et al (2012) à proposer un référentiel plus détaillé présenté dans le tableau 5. Meyer et al (2006) ont aussi établi une équation de prédiction des besoins en eau d'abreuvement de jeunes bouvillons Holstein en croissance entre 157 et $563 \mathrm{~kg}$ de poids vif. Aussi bien dans les mesures de Ménard et al (2012) que dans l'équation de Meyer et al (2006), la température ambiante est un déterminant important des besoins en eau. Dans le référentiel de Ménard et al (2012), les besoins en eau d'abreuvement sont estimés à partir de l'âge de l'animal, du type de fourrage dominant et de la température ambiante maximale. Dans l'équation de Meyer et al (2006), la température, le niveau d'ingestion, la part de fourrage dans la ration, la teneur en MS du fourrage et le poids vif sont les variables prédictives de l'eau bue. La teneur en MS n'est apparue que comme la quatrième variable prédictive de cette équation, mais les animaux n'étaient nourris qu'avec des rations à base d'ensilage de maïs.

\section{Conclusion}

Cette synthèse montre que plusieurs modèles peuvent être utilisés pour prédire les besoins en eau des vaches laitières et donne quelques outils pour choisir au mieux les équations à considérer et calculer des besoins en eau à l'échelle du troupeau laitier. Parmi les modèles que nous recommandons, le modèle de Boudon et al (2012) a montré de bonnes performances prédictives sur notre base de données de comparaison. Le modèle de Stockdale et King (1983, éq. 17) nous semble aussi intéressant étant donnés ses bonnes performances prédictives et le fait qu'il ne repose que sur

Tableau 5. Référentiel issu du suivi pendant un an dans 11 élevages laitiers commerciaux et expérimentaux, des quantités d'eau bue par les génisses et les vaches taries (en L/j/animal) selon le type de fourrage dominant dans la ration et la classe de température maximale (Ménard et al 2012).

\begin{tabular}{|c|c|c|c|c|c|c|c|c|}
\hline \multirow{2}{*}{$\begin{array}{l}\text { Classes } \\
\text { animales }\end{array}$} & \multirow{2}{*}{$\begin{array}{l}\text { Température } \\
\text { maximale }\left({ }^{\circ} \mathrm{C}\right)\end{array}$} & \multicolumn{5}{|c|}{ Type de ration $=$ fourrages dominants } & \multicolumn{2}{|c|}{ Effets $^{(4)}$} \\
\hline & & E. maïs & Foin / paille & Pâturage & $\begin{array}{l}\text { E. maïs + } \\
\text { pâturage }\end{array}$ & $\begin{array}{c}\text { Foin + } \\
\text { pâturage }\end{array}$ & $\begin{array}{c}\text { Temp. } \\
\text { max. }\end{array}$ & $\begin{array}{l}\text { Type } \\
\text { ration }\end{array}$ \\
\hline $\begin{array}{l}\text { Vaches taries, } \\
\text { génisses }>2 \text { ans }\end{array}$ & $\begin{array}{l}<20 \\
20 \text { à } 25 \\
\geq 25\end{array}$ & $\begin{array}{c}41,4(2,2)^{(1)} \\
/^{(2)} \\
/\end{array}$ & $\begin{array}{c}54,8(9,0)^{(3)} \\
62,5(9,0) \\
/\end{array}$ & $\begin{array}{l}21,7(2,6) \\
34,5(2,4) \\
45,2(2,6)\end{array}$ & $\begin{array}{l}1 \\
1 \\
1\end{array}$ & $\begin{array}{c}31,9(3,3) \\
51,4(3,8) \\
/\end{array}$ & *** & *** \\
\hline $\begin{array}{l}\text { Génisses } \\
1 \text { à } 2 \text { ans }\end{array}$ & $\begin{array}{c}<20 \\
20 \text { à } 25 \\
\geq 25\end{array}$ & $\begin{array}{c}27,2(3,3) \\
/ \\
/\end{array}$ & $\begin{array}{c}29,5(2,8) \\
33,2(3,0) \\
/\end{array}$ & $\begin{array}{l}14,8(3,0) \\
27,3(2,7) \\
34,4(3,5)\end{array}$ & $\begin{array}{c}20,3(4,1) \\
/ \\
/\end{array}$ & $\begin{array}{l}21,7(3,0) \\
35,2(4,3) \\
47,6(6,5)\end{array}$ & *** & *** \\
\hline $\begin{array}{l}\text { Génisses } \\
6 \text { mois à } 1 \text { an }\end{array}$ & $\begin{array}{l}<20 \\
20 \text { à } 25 \\
\geq 25\end{array}$ & $\begin{array}{c}19,0(2,3) \\
22,5(2,7) \\
/\end{array}$ & $\begin{array}{l}19,5(2,2) \\
25,2(3,4) \\
32,0(5,4)\end{array}$ & $\begin{array}{l}1 \\
1 \\
1\end{array}$ & $\begin{array}{l}18,6(3,9) \\
22,6(3,1) \\
31,0(4,8)\end{array}$ & $\begin{array}{l}1 \\
1 \\
1\end{array}$ & *** & NS \\
\hline $\begin{array}{l}\text { Veaux après } \\
\text { sevrage }\end{array}$ & $\begin{array}{l}<20 \\
20 \text { à } 25 \\
\geq 25\end{array}$ & $\begin{array}{l}1 \\
1 \\
1\end{array}$ & $\begin{array}{l}10,4(2,4) \\
16,7(3,2) \\
21,2(5,4)\end{array}$ & $\begin{array}{l}1 \\
1 \\
1\end{array}$ & $\begin{array}{l}1 \\
1 \\
1\end{array}$ & $\begin{array}{l}1 \\
1 \\
1\end{array}$ & $* * *$ & / \\
\hline
\end{tabular}

(1) * moyennes (et écart-type entre parenthèses) ; ${ }^{(2)} /=$ situations non rencontrées ; ${ }^{(3)}$ cellules grisées $=$ valeurs avec effectif faible $(\leq 5) ;{ }^{(4)} \mathrm{NS}=$ Non Significatif $(\mathrm{P}>0,05) ;{ }^{* * *} \mathrm{P} \leq 0,001$ 
deux paramètres à estimer. Le modèle de Holter et Urban (1992) peut aussi donner de bon résultats à condition que la température ambiante ne dépasse pas $15^{\circ} \mathrm{C}$.

Depuis 2006 (article L2224-12-5 du Code Général des Collectivités Territoriales), toutes les exploitations doivent être équipées de compteurs d'eau y compris sur les forages privés. Ceci peut permettre de détecter certaines fuites du réseau et de contrôler si les quantités d'eau d'abreuvement consommées sont suffisantes pour que l'on puisse considérer que l'abreuvement n'est pas restreint.
Pour un système d'élevage donné, les économies d'eau sur le poste d'abreuvement peuvent difficilement être réalisées sans nuire de façon immédiate à la capacité de production et au bien-être des animaux. Il est même recommandé de bien veiller à ce que les troupeaux aient toujours à disposition de l'eau de qualité nécessaire à leur abreuvement notamment pendant les périodes de fortes chaleurs. Cela n'empêche pas, dans les zones régulièrement soumises à des pénuries d'eau, de revoir l'organisation du système de production pour adapter les effectifs et les cycles de production aux périodes de pénurie en eau. Le poste de lavage du matériel de traite et des bâtiments est un poste sur lequel la marge de manœuvre est sans doute plus grande du fait de solutions techniques proposées comme le recyclage des eaux de rinçage moins chargées en lait et en produits lessiviels, l'utilisation de produit sans prélavage ou le mouillage préalable des sols et des murs (Massabie et al 2013). Rappelons que, quel que soit le poste de dépense en eau, les fuites sur le réseau de distribution d'eau peuvent constituer une source de réduction des dépenses en eau non négligeable dans certains élevages.

\section{Références}

Alais C., 1984. Science du Lait: Principe des Techniques Laitières. Edition SEPAIC, Paris, France, 814p.

Andrew S.M., Erdman R.A., Waldo D.R. 1995. Prediction of body composition of dairy cows at three physiological stages from deuterium oxide and urea dilution. J. Dairy Sci., 78, 1083-1095.

ANSES, 2010. Etat des lieux des pratiques et recommandations relatives à la qualité sanitaire de l'eau d'abreuvement des animaux d'élevage - Saisine 2008-SA-0162. ANSES, MaisonsAlfort, France, 121p.

Artaux A, Watiotienne A, Grillon F., White S., Noiret B., Cardot V., Jurjanz S., 2011. Préférences des vaches laitières pour différents types d'abreuvoirs. Renc. Rech. Rum., 18, 121.

Bannink A., Valk H., Van Vuuren A.M., 1999. Intake and excretion of sodium, potassium, and nitrogen and the effects on urine production by lactating dairy cows. J. Dairy Sci. 82, 1008-1018.

Bibby J., Toutenburg H., 1977. Prediction and improved estimation in linear model. John Wiley \& Sons, New York, USA, 202p.

Boudon A., Khelil-Arfa H., Thomas-Morel M., Banville M., Pham T.H.V., Ménard J.L., Brunschwig P., Huneau T., Coutant S., Lamy E., Faverdin P., 2012. Construction et validation d'un modèle de prédiction des besoins en eau des vaches laitières incluant l'effet de la température ambiante. Renc. Rech. Rum., 19, 177-180.

Burgos M.S., Senn M., Sutter F., Kreuzer M., Langhans W., 2001. Effect of water restriction on feeding metabolism in dairy cows. Am. J. Physiol. Regul. Integr. Comp. Physiol., 280, R418-R427.

Burgos S.A., Fadel J.G., DePeters E.J., 2007. Prediction of ammonia emission from dairy cattle manure based on milk urea nitrogen: relation of milk urea nitrogen to urine urea nitrogen excretion. J. Dairy Sci., 90, 5499-5508.

Cabrera-Estrada J.I., 2003. Le rôle de la teneur en matière séche de l'herbe sur la régulation de l'ingestion volontaire chez la vache laitière. PhD Thesis, Ecole Nationale Supérieure d'Agronomie de Rennes, Rennes, France.

Cafe L.M., Poppi D.P., 1994. The fate and behaviour of imbibed water in the rumen of cattle. J. Agric. Sci., Camb., 122, 139-144.
Cardot V., Le Roux Y., Jurjanz S., 2008. Drinking behavior of lactating dairy cows and prediction of their water intake. J. Dairy Sci., 91, 2257-2264.

Carteau B., Kerner S., Aubert C., Blondon G., Espagnol S., Massabie P., Ménard J.L., Soing P., Bonneau J., 2010. Gestion quantitative de la ressource en eau (hors irrigation). Projet CasDAR gestion durable des ressources en eau. APCA, ACTA, Chambres d'Agriculture, Instituts Techniques Agricoles, 20p.

Castle M.E., Thomas T.P., 1975. The water intake of British Friesian cows on rations containing various forages. Anim. Prod., 20, 181-189.

Corson M.S., Doreau M., 2013. Evaluation de l'utilisation de l'eau en élevage. INRA Prod. Anim., 26, 239-248.

Cunningham J.G., Klein B.G., 2007. Veterinary Physiology. Saunders-Elsevier, St-Louis, Missouri, USA, 700p.

Dahlborn K., Akerlind M., Gustafson G., 1998. Water intake by dairy cows selected for high or low milk-fat percentage when fed two forage to concentrate ratios with hay or silage. Swed. J. Agri. Res., 28, 167-176.

De Boyer des Roches A., Mounier L., Coignard M., Bareille N., Guatteo R., Veissier I., 2012. Bien-être animal en élevage : problèmes majeurs rencontrés et relation avec certaines caractéristiques des fermes. Renc. Rech. Rum., 19, 277-280.

EFSA, 2010. Scientific opinion on dietary reference values for water. EFSA Journal, 8, 1459.

Essig M., Friedlander G., 2009a. Physiologie de l'eau. In: Réanimation médicale. Boles J.M., Bollaert P.E., Jaeger A., Offenstadt G., Saulnier F., Wolff M., Zéni F. (Eds). Masson, Issy-les-Moulineaux, France, 137-141.

Essig M., Friedlander G., 2009b. Physiologie du sodium. In : Réanimation médicale. Boles J.M., Bollaert P.E., Jaeger A., Offenstadt G., Saulnier F., Wolff M., Zéni F. (Eds). Masson, Issy-les-Moulineaux, France, 141-145.

Faichney G.J., Boston R.C., 1985. Movement of water within the body of sheep fed at maintenance under thermoneutral conditions. Aust. J. Biol. Sci., 38, 85-94.

Fitzsimons J.T., 1998. Angiotensin, thirst, and sodium appetite. Physiol. Rev., 78, 583-686.
Holter J.B., Urban W.E. Jr., 1992. Water partitioning and intake prediction in dry and lactating Holstein cows. J. Dairy Sci., 75, 14721479.

Houillier P., 2009. Physiologie du potassium. In : Réanimation médicale. Boles J.M., Bollaert P.E., Jaeger A., Offenstadt G., Saulnier F., Wolff M., Zéni F. (Eds). Masson, Issy-lesMoulineaux, France, 133-137.

INRA, 1988. Alimentation des bovins, ovins et caprins. INRA Editions, Paris, France, 471p.

Jenkinson D.M., Mabon R.M., 1973. The effect of temperature and humidity on skin surface $\mathrm{pH}$ and the ionic composition of skin secretions in Ayrshire cattle. Br. Vet. J., 129, 282-295.

Khelil-Arfa H., 2012. Etude des déterminants du bilan eau d'une vache laitière et modélisation des flux journaliers. Thèse Agrocampus Ouest sous le label de l'Université Européenne de Bretagne pour obtenir le grade de Docteur d'Agrocampus Ouest.

Khelil-Arfa H., Boudon A., Maxin G., Faverdin P. 2012. Prediction of water intake and excretion flows in Holstein dairy cows under thermoneutral conditions. Animal, 6, 1662-1676.

Kume S., Nonaka K., Oshita T., Kozakai T., 2010. Evaluation of drinking water intake, feed water intake and total water intake in dry and lactating cows fed silages. Livest. Sci., 128, 46-51.

Langhans W., Rossi R., Scharrer E., 1995. Relationships between feed and water intake in ruminants. In: Ruminant Physiology: Digestion, Metabolism, Growth and Reproduction. Engelhardt W. V., Leonhard-Marek S., Breves G., Giesecke D. (Eds). Ferdinand Enke Verlag, Stuttgart, Allemagne, 199-216.

Little W., Shaw S.R., 1978. A note on the individuality of the intake of drinking water by dairy cows. Anim. Prod., 26, 225-227.

Macfarlane W.V., Howard B., 1972. Comparative water and energy economy of wild and domestic mammals. Proc. Symp. Zool. Soc., 31, 261-296.

Maia A.S.C., DaSilva R.G., Battiston Loureiro C.M., 2005a. Sensible and latent heat loss from the body surface of Holstein cows in a tropical environment. Int. J. Biometeorol., 50, 17-22. 
Maia A.S.C., DaSilva R.G., Battiston Loureiro C.M., 2005b. Respiratory heat loss of Holstein cows in a tropical environment. Int. J. Biometeorol., 49, 332-336.

Massabie P., Aubert C., Ménard J.L., Roy H., Boulestreau-Boulay A.L., Dubois A., Dezat E., Dennery G., Roussel P., Martineau C., Brunschwig P., Thomas J., Quillien J.P., Briand P., Coutant S., Fulbert L., Huneau T., Lowagie S., Magnière J.P., Nicoud M., Piroux D., Boudon A., 2013. Maîtrise des consommations d'eau en élevage : élaboration d'un référentiel, identification des moyens de réduction, construction d'une démarche de diagnostic. Innovations Agronomiques, (sous presse).

McKinley M.J., Johnson A.K., 2004. The physiological regulation of thirst and fluid Intake. News Physiol. Sci., 19, 1-6.

Ménard J.L., Lepesme M., Brunschwig P., Coutant S., Fulbert L., Huneau T., Libeau J., Lowagie S., Magnière J.P., Nicoud M., Piroux D., Boudon A., 2012. Evaluation de la consommation en eau en élevage bovins laitiers et mise au point d'un référentiel simplifié de l'abreuvement des vaches, génisses et veaux après sevrage. Renc. Rech. Rum., 19, 173-176.

Meschy F., 2010. Nutrition minérale des ruminants. Editions Quae, Versailles, France, 208p.

Meyer U., Everinghoff M., Gõdeken D., Flachowsky G., 2004. Investigations on the water intake of lactating dairy cows. Livest. Prod. Sci., 90, 117-121.
Meyer U., Stahl W., Flachowsky G., 2006. Investigations on the water intake of growing bulls. Livest. Sci., 103, 186-191.

Murphy M.R., 1992. Water metabolism of dairy cattle. J. Dairy Sci., 75, 326-333.

Murphy M.R., Davis C.L., McCoy G.C., 1983. Factors affecting water consumption by Holstein cows in early lactation. J. Dairy Sci., 66, 35-38.

NRC, 2001. Nutrients requirements of dairy cattle. Seventh revised edition. National Academy Press, Washington DC, USA, 381p.

Pachauri R.K., Reisinger A., 2007. Climate Change 2007: Synthesis Report. 104p. IPCC, Genève, Suisse, 103p.

Paquay R., De Baere R., Lousse A., 1970a. Statistical research on the fate of water in the adult cow. I. Dry cows. J. Agric. Sci., Camb., 74, 423-432.

Paquay R., De Baere R., Lousse A., 1970b. Statistical research on the fate of water in the adult cow. II. The lactating cow. J. Agric. Sci., Camb., 75, 251-255.

Pinheiro Machado Filho L.C., Teixeira D.L., Weary D.M., Keyserlingk M.A.G. von, Hötzel M.J., 2004. Designing better water troughs: dairy cows prefer and drink more from larger troughs. App. Anim. Behav. Sci., 89, 185193.

Silanikove N., 1989. Interrelationships between water, food and digestible energy intake in desert and temperate goats. Appetite, 12, 163-170.

Silanikove N., 2000. Effects of heat stress on the welfare of extensively managed domestic ruminants. Livest. Prod. Sci., 67, 1-18.

Steinfeld H., Gerber P., Wassenaar T., Castel V., Rosales M., De Haan C., 2006. Livestock's long shadow. Environmental issues and options. FAO, Rome, Italie, 390p.

Stockdale C.R., King K.R., 1983. A note on some of the factors that affect the water consumption of lactating dairy cows at pasture. Anim. Prod., 36, 303-306.

Teixeira, D.L., Hötzel M.J., Machado Filho L.C.P., 2006. Designing better water troughs: 2. Surface area and height, but not depth, influence dairy cows' preference. App. Anim. Behav. Sci., 96, 169-175.

Welfare Quality®, 2009. Welfare Quality® assessment protocol for cattle (Fattening Cattle, Dairy Cows, Veal Calves). Welfare Quality® Consortium: Lelystad, Pays-Bas, 182p. (http://www.welfarequalitynetwork.net/downloadattachment/45627/21650/Cattle\%20Protocol $\% 20$ without \%20Veal\%20Calves.pdf)

Woodford S.T., Murphy M.R., Davis C.L., 1984a. Water dynamics of dairy cattle as affected by initiation of lactation and feed intake. J. Dairy Sci., 67, 2336-2343.

Woodford S.T., Murphy M.R., Davis C.L., Holmes K.R., 1984b. Ruminal bypass of drinking water in lactating cows. J. Dairy Sci., $67,2471-2474$.

\section{Résumé}

L'eau d'abreuvement est un besoin incompressible des élevages laitiers. Elle représente un coût non négligeable et sa restriction conduit immédiatement à des baisses de performances et à une altération du bien-être des troupeaux. Le bilan hydrique de l'animal est équilibré grâce à la régulation de l'excrétion rénale d'eau et des volumes d'eau bue en réponse à des consignes de pression osmotique plasmatique et de volume sanguin. De nombreuses équations de prédiction des besoins en eau des vaches laitières ont été publiées. La teneur en matière sèche de la ration ressort comme le premier paramètre prédictif des volumes d'eau bue parce qu'elle détermine la part relative de l'eau provenant de l'aliment et bue dans l'eau totale ingérée. La quantité de matière sèche ingérée, les teneurs en sodium, potassium et azote de la ration ainsi que la température ambiante sont les principaux paramètres prédictifs de l'eau totale ingérée. La comparaison des erreurs de prédiction des équations des besoins en eau des vaches laitières sur un jeu de données commun montre des performances très hétérogènes. Les critères de choix des équations doivent être la nature des paramètres prédictifs disponibles mais aussi les conditions dans lesquelles elles ont été établies. Les équations de prédiction des besoins en eau des vaches laitières ont souvent été établies à partir de mesures individuelles et leur application sur un troupeau disposant d'abreuvoirs à niveau constant peut entrainer un biais de prédiction. Quelques pistes sont évoquées pour quantifier les besoins en eau des génisses.

\section{Abstract}

\section{Watering requirements of dairy herds: physiological determinisms and quantification}

Drinking water is an incompressible need of dairy farms. It represents a significant cost and, an immediate consequence of its restriction is a decrease in milk yield and an alteration of animal welfare. Animal water balance is maintained thanks to regulation of renal excretion of water and thirst in responses to set points related to plasmatic osmotic pressure and blood volume. Numerous predictive equations for water requirements of dairy cows have been published. Diet dry matter content is the first determinant of drinking water volume because it determines the relative part of feed water and drinking water in the total amount of water intake. Dry matter intake, diet contents of sodium, potassium and nitrogen and ambient temperature are the main determinants of total water intake. The comparison of the prediction error of the predictive equations on a common data set showed contrasted performances. The choice of an equation should be based on the nature of the available predictors and the conditions in which it was established. Given that most of the predictive equations for dairy cow water requirements are established from individual measurements, their application to a herd watered through constant trough level requires adjustments. Some elements are also given in the paper to predict the water requirements of heifers. 